\title{
New perspectives on the plant PARP family: Arabidopsis PARP3 is inactive, and PARP1 exhibits predominant poly (ADP-ribose) polymerase activity in response to DNA damage
}

Zongying Gu, Weiyang Pan, Wei Chen, Qichao Lian, Qiao Wu, Zeyu Lv, Xuan Cheng and Xiaochun Ge*

\begin{abstract}
Background: Poly (ADP-ribosyl) ation (PARylation) is an important posttranslational modification that regulates DNA repair, gene transcription, stress responses and developmental processes in multicellular organisms. Poly (ADP-ribose) polymerase (PARP) catalyzes PARylation by consecutively adding ADP-ribose moieties from NAD ${ }^{+}$to the amino acid receptor residues on target proteins. Arabidopsis has three canonical PARP members, and two of these members, AtPARP1 and AtPARP2, have been demonstrated to be bona fide poly (ADP-ribose) polymerases and to regulate DNA repair and stress response processes. However, it remains unknown whether AtPARP3, a member that is highly expressed in seeds, has similar biochemical activity to that of AtPARP1 and AtPARP2. Additionally, although both the phylogenetic relationships and structural similarities indicate that AtPARP1 and AtPARP2 correspond to animal PARP1 and PARP2, respectively, two previous studies have indicated that AtPARP2, and not AtPARP1, accounts for most of the PARP activity in Arabidopsis, which is contrary to the knowledge that PARP1 is the predominant PARP in animals.
\end{abstract}

Results: In this study, we obtained both in vitro and in vivo evidence demonstrating that AtPARP3 does not act as a typical PARP in Arabidopsis. Domain swapping and point mutation assays indicated that AtPARP3 has lost $\mathrm{NAD}^{+}$-binding capability and is inactive. In addition, our results showed that AtPARP1 was responsible for most of the PARP enzymatic activity in response to the DNA damage-inducing agents zeocin and methyl methanesulfonate (MMS) and was more rapidly activated than AtPARP2, which supports that AtPARP1 remains the predominant PARP member in Arabidopsis. AtPARP1 might first become activated by binding to damaged sites, and AtPARP2 is then poly (ADP-ribosyl) ated by AtPARP1 in vivo.

Conclusions: Collectively, our biochemical and genetic analysis results strongly support the notion that AtPARP3 has lost poly (ADP-ribose) polymerase activity in plants and performs different functions from those of AtPARP1 and AtPARP2. AtPARP1, instead of AtPARP2, plays the predominant role in PAR synthesis in both seeds and seedlings. These data bring new insights into our understanding of the physiological functions of plant PARP family members.

Keywords: Arabidopsis thaliana, DNA damage response, ADP-ribosylation, Posttranslational modification, Poly (ADP-ribose) polymerase

\footnotetext{
* Correspondence: xcge@fudan.edu.cn

State Key Laboratory of Genetic Engineering, Department of Biochemistry and Molecular Biology, School of Life Sciences, Fudan University, Shanghai 200438, China
}

(c) The Author(s). 2019 Open Access This article is distributed under the terms of the Creative Commons Attribution 4.0 International License (http://creativecommons.org/licenses/by/4.0/), which permits unrestricted use, distribution, and reproduction in any medium, provided you give appropriate credit to the original author(s) and the source, provide a link to the Creative Commons license, and indicate if changes were made. The Creative Commons Public Domain Dedication waiver (http://creativecommons.org/publicdomain/zero/1.0/) applies to the data made available in this article, unless otherwise stated. 


\section{Background}

Poly (ADP-ribose) polymerase (PARP), the enzyme that catalyzes the protein poly (ADP-ribosyl) ation (PARylation) modification, exists widely in different eukaryotes except yeast [1]. PARP transfers ADP-ribose units from the substrate $\mathrm{NAD}^{+}$to acceptor amino acids on target proteins to produce long linear or branched chains of poly (ADP-ribose) (PAR), and these units can then be removed by a hydrolysis enzyme, poly (ADP-ribose) glycohydrolase (PARG) $[1,2]$. Modified target proteins include the enzyme itself, histones, DNA repair proteins, transcription factors, chromatin modulators $[2,3]$ and proteins involved in defense response [4]. Due to its highly negative charges, PAR largely affects the protein-protein interaction features of target proteins. PAR might act as a protein-binding scaffold to recruit other proteins for the formation of large complexes [2]. Proteins can bind to PARylated proteins through their PAR-binding motifs [2, 5]. PARP family members have been extensively studied in humans because they are implicated in multiple pathogenesis processes $[6,7]$. PARP inhibitors for use in research or medicine have then been developed, and some of these mimic the structure of $\mathrm{NAD}^{+}$ and act as competitive inhibitors, such as 3-aminobenzamide (3-AB) [5, 8-10]. Seventeen members in humans are involved in various physiological processes, such as DNA repair, cell death, transcriptional regulation, energy metabolism, and chromatin remodeling $[2,11,12]$.

The functions of the PARP family in plants are much less well understood. PARP activities have been identified in the nuclei of different species, such as wheat [13], pea [13], soybean [14], tobacco [15, 16], maize [13, 17] and Arabidopsis [17]. To date, three types of PARPs with different molecular masses and structural architectures have been found in plants. In Arabidopsis, these PARPs are named AtPARP1, AtPARP2 and AtPARP3 [18, 19]. AtPARP1 and AtPARP2 have auto-PARylation activity in vitro and in vivo [17, 20-22], and AtPARP2 is the predominant PARP in DNA damage and immune responses [22], but whether AtPARP3 has PARP activity remains elusive. AtPARP1 and AtPARP2 have been shown to regulate genotoxic $[23,24]$, biotic and abiotic stress responses $[4,10,14,15,20,22,25,26]$, as well as leaf and root development $[9,21,27,28]$. However, a recent study indicated that double and triple mutants of PARP family members have no phenotypes under abiotic stresses or under biotic stress stimulated by the microbe-associated molecular pattern (MAMP) flg22 [29], and as a result, the in vivo functions of PARPs in both biotic and abiotic stresses are controversial. AtPARP3 is highly expressed in seeds, where both AtPARP1 and AtPARP2 are almost undetectable [30, 31]. It is reported that AtPARP3 is involved in the maintenance of seed viability during seed storage [27,
30], therefore, AtPARP3, instead of AtPARP1 and AtPARP2, was hypothesized to be the major enzyme catalyzing PARylation and responsible for DNA repair during seed germination [30, 31].

PARP proteins are classified by the PARP signature consisting of a central six-stranded $\beta$ sheet that binds and catalyzes the decomposition of $\operatorname{NAD}^{+}[11,19,32]$. A catalytic core motif histidine-tyrosine-glutamic acid (H-Y-E) triad has been found in all active PARP proteins and is involved in $\mathrm{NAD}^{+}$binding. Based on the domain architecture of the proteins, AtPARP1 and AtPARP2 resemble human HsPARP1 and HsPARP2, respectively [18, 19], whereas AtPARP3 has no counterpart in humans.

Whether a protein exhibits known biochemical activity is important for explaining its physiological role. To understand the function of AtPARP3 in seeds, it is critical to determine its enzymatic activity. In addition, elucidating the functional relationships among members of this protein family will also help us understand the family's evolution and differentiation. In this study, through a combination of biochemical and genetic approaches, we demonstrated that AtPARP3 exhibits no poly (ADP-ribosyl) ation activity in vitro and in vivo. We also found that AtPARP1 exhibits predominant PARP enzymatic activity relative to other family members in both seeds and seedlings when stimulated by DNA breaks. This result differs from the previous finding that AtPARP2 is the predominant PARP member in DNA damage response but is consistent with the role of its animal PARP1 counterpart, which accounts for most of the intracellular PARP activity under genotoxic stress. Our results shed new light on the nature of the plant PARP family.

\section{Results}

\section{Domain architectures and phylogenetic relationships of} PARP $1 / 2 / 3$

AtPARP1 is very similar to HsPARP1 in terms of its domain architecture (Additional file 1: Figure S1A). HsPARP1 is thought to be the founding member of the PARP family in humans $[1,3,11]$. Both HsPARP1 and AtPARP1 have five important domains with known functions arranged from the $\mathrm{N}$ to the $\mathrm{C}$ terminus: three $\mathrm{N}$-terminal zinc fingers responsible for DNA damage detection, the BRCA-1 C-terminus (BRCT) domain for phospho-protein binding, the WGR domain with the conserved Trp-Gly-Arg (WGR) motif for nucleic acid binding, the PARP regulatory domain (PRD) or helical subdomain (HD) believed to regulate PAR-branching, and the C-terminal characteristic PARP domain with catalytic activity [3, 33] (Additional file 1: Figure S1A). Both HsPARP2 and AtPARP2 have no zinc fingers or BRCT domain but do have two SAF/Acinus/PIAS motif (SAP) domains in the $\mathrm{N}$-terminal region that confer 
DNA-binding activity [11, 19, 32]. HsPARP3 has only three domains, i.e., the WGR, PRD/HD and PARP domains, and catalyzes mono (ADP-ribosyl) ation (mART), which attaches only one ADP-ribose unit to the target protein [34], whereas AtPARP3 has a long N-terminal region with unknown functions and bears BRCT, WGR, $\mathrm{PRD} / \mathrm{HD}$ and the PARP catalytic domain.

The sequence analysis revealed that both AtPARP1 and AtPARP2 have a typical H-Y-E catalytic triad, whereas AtPARP3 has an alternative histidine-valineglutamic acid (C-V-E) triad in its catalytic core (Additional file 1: Figure S1B). To understand the evolutionary relationships of the PARP family in different species, we constructed a phylogenetic tree using PARP1/2/3 homologs identified from twentyeight organisms, including eighteen angiosperms, two gymnosperms, one lycopod, one moss, four metazoans and two fungi, using the PARP1 subfamily as an outgroup (Additional file 2: Figure S2). The phylogenetic tree topology revealed that the PARP1/ $2 / 3$ subfamilies form two clades in eukaryotes, each with metazoan members, which suggests that the clade containing plant PARP2 and the clade with plant PARP1/3 originated from gene duplications before the common ancestor of eukaryotic organisms. The close homologs of plant PARP1 and PARP3 form separate phylogenetic groups, each involving lycopod and moss members, which indicates that these resulted from duplications before the divergence of extant land plants but after the separation of plants from metazoans and fungi.

\section{AtPARP3 does not show auto-ADP-ribosylation activity in vitro}

Because the activity of AtPARP3 is unknown, we mainly focused on AtPARP3. We first examined the biochemical activity of recombinant AtPARP3 protein expressed in E. coli. To avoid the possible influence of a fusion tag on protein function, we used two different expression vectors, pGEX-4 T-1 and pET$32 \mathrm{a}(+)$, which carry a glutathione S-transferase (GST) and a thioredoxin and histidine (TRXH) tag fused to the $\mathrm{N}$ terminus of the recombinant protein, respectively. These two tags reportedly facilitate the correct folding of the target proteins, particularly the cysteine-rich proteins. AtPARP1 was expressed by the pET-32a(+) vector as a positive control for the activity assay because it shows stronger in vitro activity than AtPARP2, as demonstrated in our previous studies [21]. The tag proteins were also expressed using the empty vectors for use as negative controls. We assessed their activities through a standard PARP activity assay based on the auto-modification feature of PARP proteins [35]. A sensitive ADP-ribose detection reagent that can detect both mono- and poly-ADP-ribose, called anti-pan-ADP-ribose binding reagent, was used to detect the ADP-ribose moiety on proteins (based on the certificate of analysis provided by Merck). It has been successfully used in a prior study on humans [36]. As shown in Fig. 1a, a strong PAR signal was generated by AtPARP1 within $1 \mathrm{~min}$, and the band exhibited an upward smear typical of poly (ADP-ribosyl) ation, consistent with previous observations that PARP1 is a robust enzyme synthesizing PAR in seconds [35, 37]. The signal was abolished by the PARP inhibitor $3-\mathrm{AB}$, which indicated that it was a real PAR signal. However, no ADP-ribose signal was detected in the samples containing either GSTAtPARP3 or TRXH-AtPARP3. To exclude the possibility that AtPARP3 might need a longer reaction time, we extended the incubation time to $30 \mathrm{~min}$, and no PAR signal was detected (Fig. 1b).

\section{The AtPARP3 catalytic domain exhibits no activity}

The PARP catalytic domain is important for PARP activity. The N-terminal DNA-binding domain can interact with and activate the C-terminal PARP catalytic domain after binding to DNA [33, 35]. Although AtPARP3 has BRCT, WGR, PRD/HD and PARP catalytic domains, it has no recognized DNA-binding domain in the $\mathrm{N}$-terminal region (Additional file 1: Figure $\mathrm{S} 1$ ); in addition, a C-V-E motif replaces the classical $\mathrm{H}$ Y-E triad in the catalytic core. To assess whether this domain has function in catalyzing PAR formation, we exchanged the catalytic domain of AtPARP1 with that of AtPARP3, as shown in Fig. 2a. The chimera proteins AtP1-P3 and AtP3-P1 were produced in the same expression system as AtPARP1 and AtPARP3. In contrast to the strong activity of AtPARP1, the AtPARP1 protein carrying the AtPARP3 catalytic domain (AtP1-P3) showed no PARP activity (Fig. 2b), which indicated that the AtPARP3 catalytic domain failed to form PAR even in the presence of other functional domains from AtPARP1.

However, the chimera PARP3 protein AtP3-P1, in which most domains of AtPARP3 were retained and only the catalytic domain was replaced by that of AtPARP1 (Fig. 2a), showed constitutive activity, and the PAR signal was detected at all time points, even at the time point " 0 " in the absence of exogenously supplemented $\mathrm{NAD}^{+}$and DNA (Fig. 2b). 3-AB only slightly reduced the signal, which indicated that the signal had been produced in $E$. coli cells prior to purification, and $\mathrm{NAD}^{+}$and DNA supplementation during the assay only mildly enhanced its activity. These results indicated that the catalytic activity of the AtPARP1 domain in AtPARP3 was constitutively "switched on." 


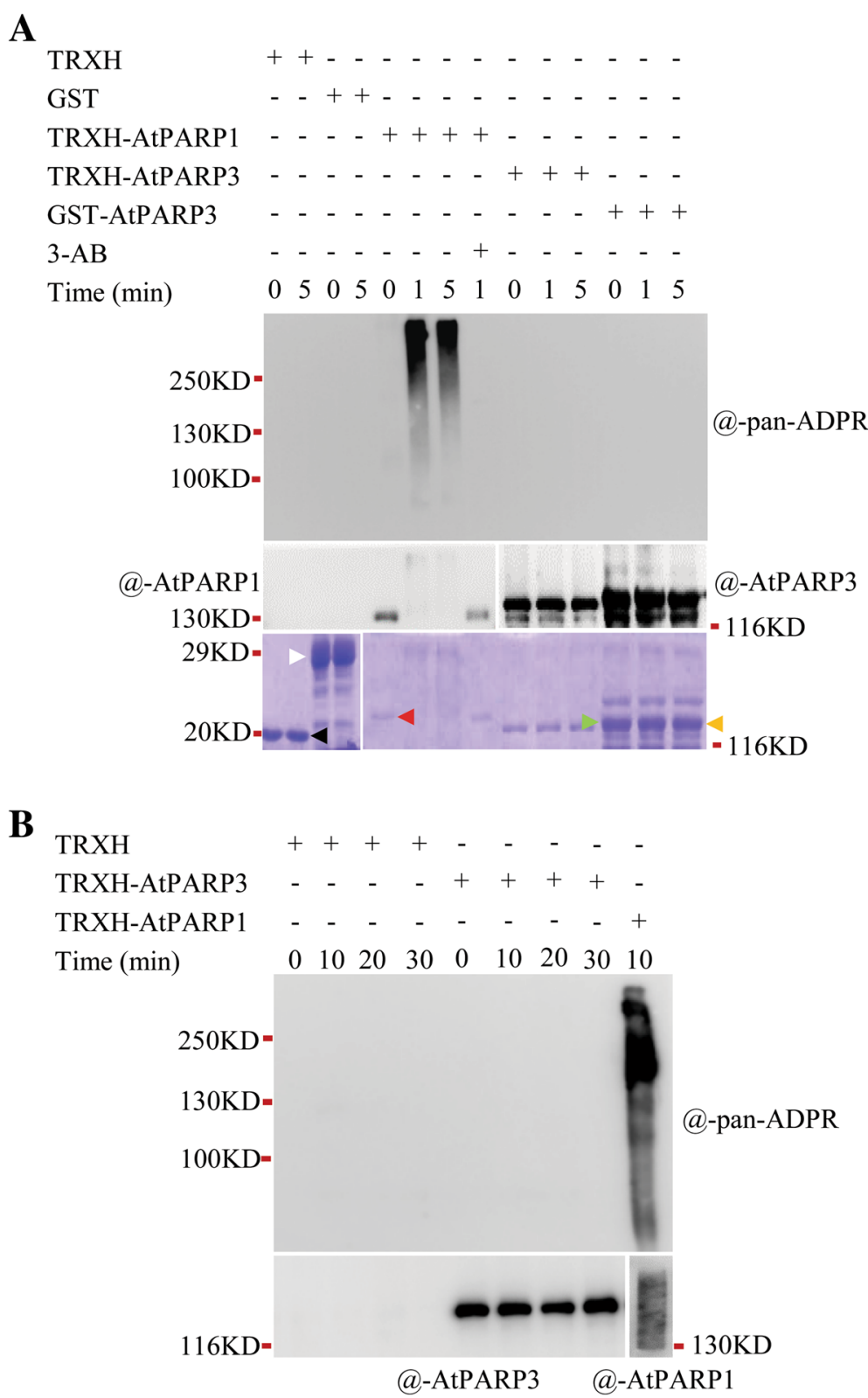

Fig. 1 Determination of the enzymatic activity of AtPARP3. a Recombinant AtPARP3 activity determination. The purified proteins were incubated with $500 \mathrm{nM}$ broken DNA and $1 \mathrm{mM} \mathrm{NAD}^{+}$at $25^{\circ} \mathrm{C}$ for different time periods with or without $20 \mathrm{mM}$ 3-AB. After electrophoresis, immunoblotting was performed with anti-pan-ADPR reagent or other internal control antibodies. The tag proteins, TRXH and GST, expressed by the empty vectors pET32a(+) and pGEX-4T-1, respectively, were used as the negative controls for the assay. The Coomassie blue-stained SDS-PAGE gel in the bottom panel shows the loading amounts of TRXH (black arrow), GST (white arrow), TRXH-AtPARP1 (red arrow), TRXH-AtPARP3 (green arrow), and GST-AtPARP3 (orange arrow). b Activity determination under extended time periods. The purified proteins were incubated with $500 \mathrm{nM}$ broken DNA and $1 \mathrm{mM} \mathrm{NAD}^{+}$at $25^{\circ} \mathrm{C}$ for different time periods. The poly (ADP-ribosyl) ated proteins were separated on an SDS-PAGE gel and detected by anti-pan-ADPR reagent (the upper panel) and anti-AtPARP3 and anti-AtPARP1 antibodies, respectively

Other structural elements beyond the catalytic triad also determine activity

To understand whether the loss of activity of AtPARP3 is solely due to the change in "H-Y" of the catalytic triad, we mutated the "H-Y" of AtPARP1 to "C-V" (AtPARP1M) by point mutation and changed the " $\mathrm{C}-\mathrm{V}$ " of AtPARP3 back to "H-Y" (AtPARP3M) (Fig. 3a), and then examined the activities of the AtPARP1 and AtPARP3 mutant proteins as well as those of their wild-type controls. No PAR signal was observed for either the AtPARP1M or AtPARP3M proteins (Fig. $3 \mathrm{~b}$ and c), which indicated that mutation of the first two amino acids of H-Y-E in AtPARP1 is sufficient to eliminate the PARP enzymatic activity; however, $\mathrm{H}-\mathrm{Y}-\mathrm{E}$ is not the only important motif for determining the 


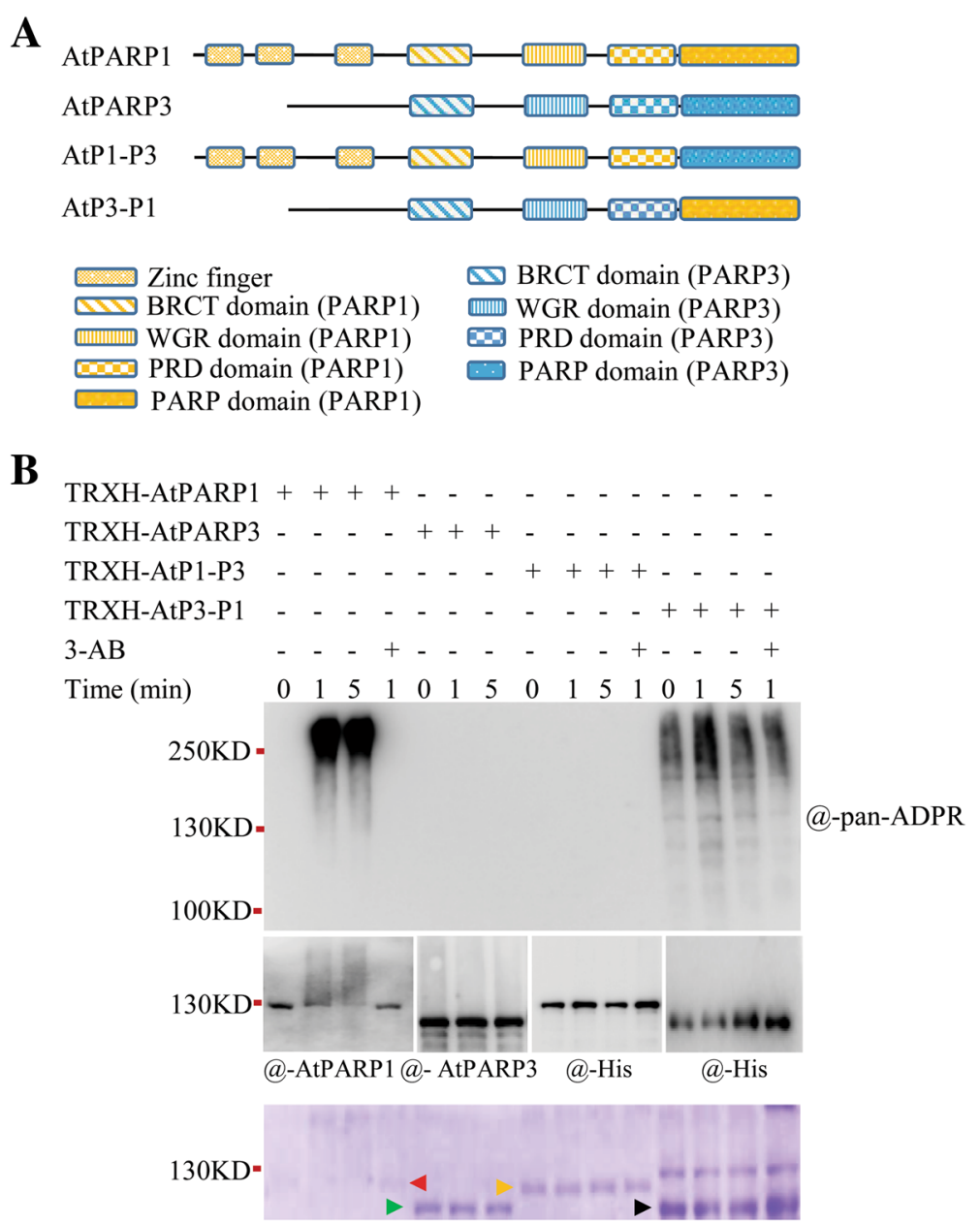

Fig. 2 Activity determination of recombinant proteins with domain swapping. a Schematic diagram of the domain swapping experiments between AtPARP1 and AtPARP3. Different boxes show different domains. $\mathbf{b}$ Activity determination of the domain-exchanged proteins. The purified proteins were incubated with $500 \mathrm{nM}$ broken DNA and $1 \mathrm{mM} \mathrm{NAD}$ at $25^{\circ} \mathrm{C}$ for different time periods with or without $20 \mathrm{mM} 3-\mathrm{AB}$ and then detected by immunoblotting using different antibodies. The arrows in the Coomassie blue-stained SDS-PAGE gel indicate the recombinant proteins TRXH-AtPARP1 (red), TRXH-AtPARP3 (green), TRXH-AtP1-P3 (orange), and TRXH-AtP3-P1 (black). Anti-His antibody was used to detect the expressed TRXH-AtP1-P3 and TRXH-AtP3-P1 proteins

activity of AtPARP3 because even a typical H-Y-E triad failed to regenerate the PARP activity in AtPARP3.

Several other motifs are also considered to be important for PARP activity [31], and they varied in AtPARP3. In addition to the catalytic triad motif, AtPARP3 carried altered motifs 1 and 2 in the $\mathrm{NAD}^{+}$fold (Additional file 1: Figure S1B). Histine-glycine-serine (H-G-S) in motif 1 and tyrosine-phenylalanine-alanine (Y-F-A) in motif 2 were replaced by cysteine-glycine-serine (C-G-S) and valine-phenylalanine-alanine (V-C-S), respectively, in AtPARP3, and these two motifs might provide a microenvironment for $\mathrm{NAD}^{+}$binding.

\section{AtPARP3 loses the ability to bind to NAD ${ }^{+}$}

Substrate recognition is the prerequisite for an enzyme to perform activity. To understand the possible structural differences among Arabidopsis PARP enzymes, we first simulated the structures of Arabidopsis AtPARP1 and AtPARP3 using the resolved crystal structures of HsPARPs as models. Representative images illustrating the binding of the PARP catalytic domain (green) to the $\mathrm{NAD}^{+}$molecule (white) are presented in Fig. 4a. The results indicated that the $\mathrm{C}$ and $\mathrm{V}$ residues in AtPARP3 failed to correctly orientate the $\mathrm{NAD}^{+}$molecule relative to those of human and Arabidopsis PARP1. To investigate whether AtPARP3 retains the capability of binding to $\mathrm{NAD}^{+}$, we calculated the binding affinities between PARP proteins and $\mathrm{NAD}^{+}$molecule using AutoDock software [39]. The binding affinity of AtPARP3 to $\mathrm{NAD}^{+}$was significantly lower than those of other known active PARP proteins. Among the PARP 
A

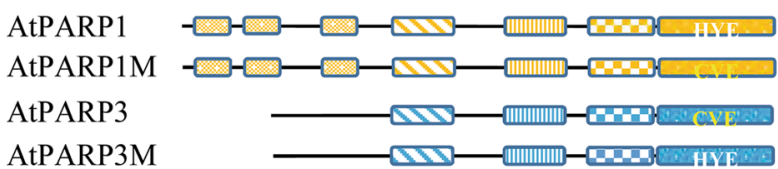

AtPARP3M

-

\section{AtPARP1 PARP domain $\because$ AtPARP3 PARP domain}

B

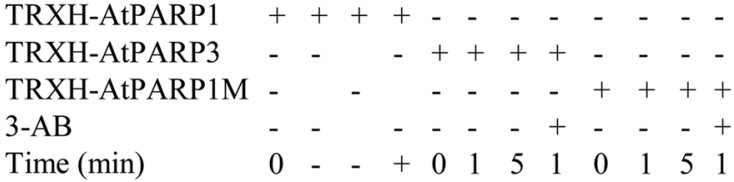

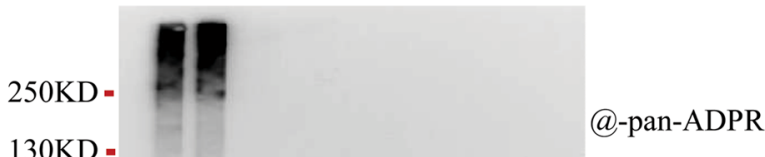

$130 \mathrm{KD}=$

@-pan-ADPR

$100 \mathrm{KD}=$

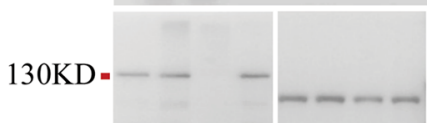

@-AtPARP1@-AtPARP3@-AtPARP1

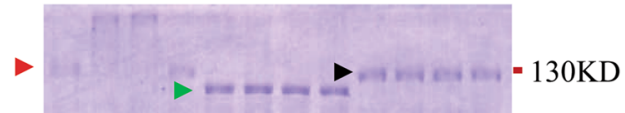

C

$\begin{array}{lllllllllllll}\text { TRXH-AtPARP1 } & + & + & + & + & - & - & - & - & - & - & - & - \\ \text { TRXH-AtPARP3 } & - & - & - & - & + & + & + & + & - & - & - & - \\ \text { TRXH-AtPARP3M } & - & - & - & - & - & - & - & - & + & + & + & + \\ \text { 3-AB } & - & - & - & + & - & - & - & + & - & - & - & + \\ \text { Time (min) } & 0 & 1 & 5 & 1 & 0 & 1 & 5 & 1 & 0 & 1 & 5 & 1\end{array}$

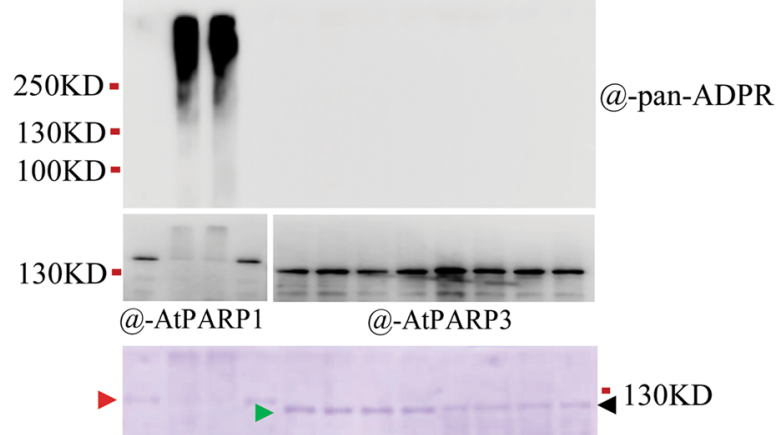

Fig. 3 Enzymatic activity of point mutation proteins. a Schematic diagrams of the proteins with point mutations. The residues in the catalytic triad are given in the PARP domain. $\mathbf{b}$ The mutated AtPARP1 protein has no catalytic activity. For TRXH-AtPARP1M, the catalytic triad in AtPARP1 was changed from a normal H-Y-E to C-V-E. c Back mutation of the catalytic triad in AtPARP3 cannot recover its activity. For TRXH-AtPARP3M, the catalytic triad in AtPARP3 was changed from C-V-E to a normal H-Y-E. The purified proteins were incubated with $500 \mathrm{nM}$ DNA and 1 mM NAD at $25^{\circ} \mathrm{C}$ for different time periods with or without $20 \mathrm{mM} 3-\mathrm{AB}$. After the reaction, the proteins were analyzed by immunoblot using different antibodies. The arrows in the Coomassie blue-stained SDS-PAGE gel indicate the recombinant proteins TRXH-AtPARP1 (red arrow), TRXH-AtPARP3 (green arrow), TRXH-AtPARP1M or TRXH-AtPARP3M (black arrow)

proteins, AtPARP1, AtPARP2, HsPARP1 and HsPARP2 were verified as poly (ADP-ribose) polymerases; HsPARP3 is a mono-ADP-transferase; and HsPARP5a and HsPARP5b mediate oligo (ADP- ribosyl) ation [32, 40]. All of these proteins can bind to $\mathrm{NAD}^{+}$. The lower binding affinity between AtPARP3 and $\mathrm{NAD}^{+}$implies that AtPARP3 might have lost the capacity to bind to NAD ${ }^{+}$. 


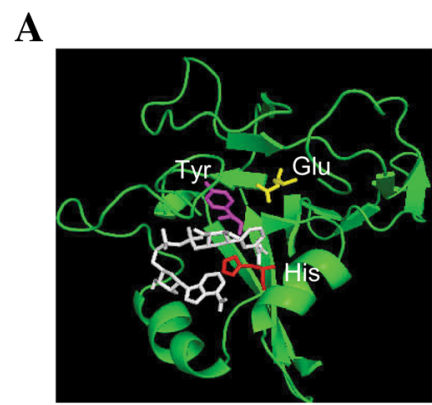

HsPARP1

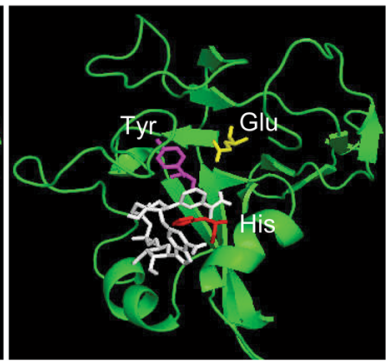

AtPARP1

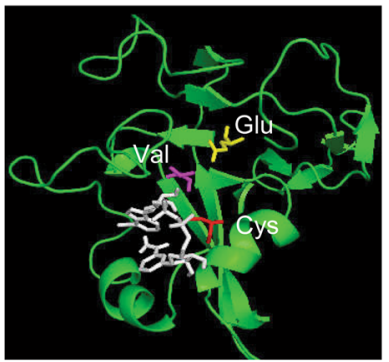

AtPARP3

B

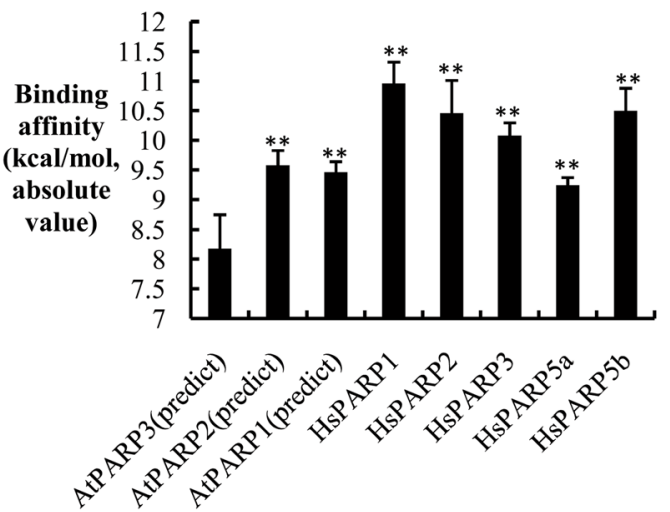

Fig. 4 Binding affinity calculations between PARPs and NAD ${ }^{+}$. a Molecular docking simulation of PARPs and NAD ${ }^{+}$. Representative images of binding between the PARP catalytic domain (green) and $\mathrm{NAD}^{+}$molecule (white) analyzed using AutoDock software. The histidine (cysteine in AtPARP3), tyrosine (valine in AtPARP3) and glutamate residues of the H-Y-E (C-V-E in AtPARP3) triad motif are labeled in red, magenta and yellow, respectively. The HsPARP1 structure was extracted from a previously published crystal structure (PDB ID: 1UK1). The AtPARP1 and AtPARP3 structures were generated using the homology-based protein structure prediction software Phyre2 [38]. b Binding affinities generated by in silico molecular docking. For each protein, the molecular docking experiment was repeated five times with the same parameter settings. The average from the top binding affinity from the various time point was used to evaluate the protein's binding affinity to the $\mathrm{NAD}^{+}$molecule. The structural information for human HsPARP1, HsPARP2, HsPARP3, HsPARP5a and HsPARP5b was downloaded from PDB, and the structures of Arabidopsis PARPs were modeled using Phyre2. Significant differences were determined using Student's t-test. ${ }^{* *} P<0.01$

To examine the binding activity of $\mathrm{NAD}^{+}$to AtPARP3, we spotted the purified proteins onto a polyvinylidene fluoride (PVDF) membrane, using the irrelevant protein bovine serum albumin (BSA) and the fusion tag protein TRXH as negative controls, and incubated the membrane with biotinylated $\mathrm{NAD}^{+}$. If $\mathrm{NAD}^{+}$bound to the proteins on the membrane, the biotin tag on $\mathrm{NAD}^{+}$would allow detection of the $\mathrm{NAD}^{+}$signal by streptavidin/HRP (Fig. 5a). An inner control anti-His antibody was used to visualize the amount of protein spotted on the membrane (Fig. 5b). The results showed that the active enzyme TRXHAtPARP1 and the chimera TRXH-AtP3-P1 protein were able to bind to $\mathrm{NAD}^{+}$, whereas the protein with the AtPARP3 catalytic domains TRXH-AtPARP3 and TRXH-AtP1-P3 could not. Moreover, the inactive protein TRXH-AtPARP3M also showed $\mathrm{NAD}^{+}$-binding activity, which suggested that the reverse mutation of $\mathrm{C}-\mathrm{V}-\mathrm{E}$ to $\mathrm{H}-\mathrm{Y}-\mathrm{E}$ recovered the $\mathrm{NAD}^{+}$-binding activity of AtPARP3 but failed to recover its PAR- generating activity, and this finding further supported the notion that other motifs around the H-Y-E triad are also important for PARP enzymatic activity. Surprisingly, the AtPARP1 protein with the H-Y-E to C$\mathrm{V}$-E mutation still maintained $\mathrm{NAD}^{+}$-binding activity even though it was inactive, but the underlying molecular basis remains unknown.

\section{AtPARP3 is not responsible for PAR formation in seeds}

PARP modifies itself and other proteins by PARylation; thus, the PAR level in vivo reflects the cellular PARP activity directly. To investigate whether AtPARP3 is physiologically active, comparison of the PAR levels in wild-type plants and loss-of-function mutants of AtPARP3 is crucial. By examining the PAR levels in parp3 mutants and in wild-type plants, we could determine whether AtPARP3 is active in vivo.

We ordered T-DNA insertion mutants of AtPARP3 from TAIR and named them parp3-1 and parp3-2. Quantitative RT-PCR (RT-qPCR) and immunoblotting 


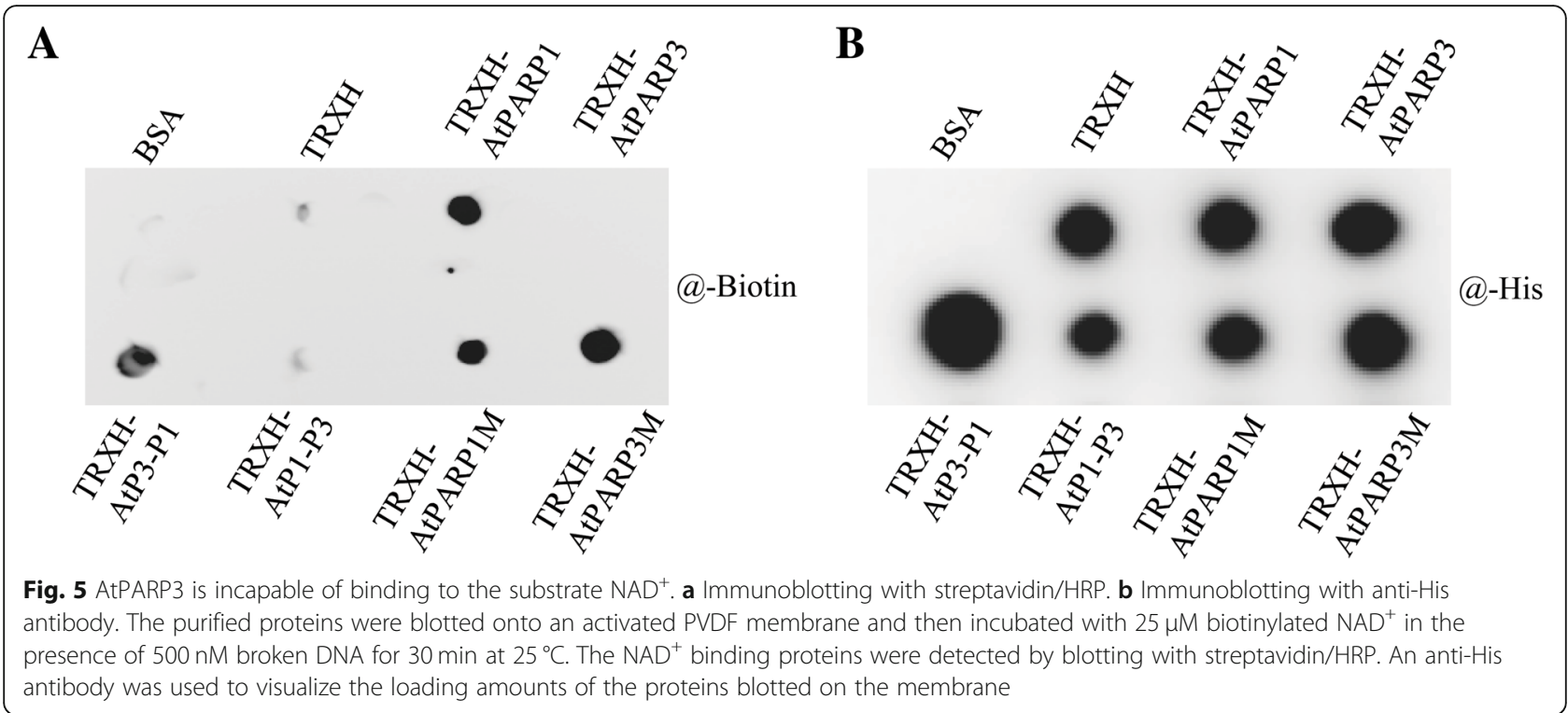

analyses confirmed that they were both null mutants (Additional file 3: Figure S3A-C). We also included multiple mutants of both AtPARP1 and AtPARP2 for comparison. For each gene, at least two different mutants were used, and all these mutants have been used in other studies [4, 20, 22-24, 27, 29]. The T-DNA insertion sites of the mutants for the AtPARP1 and AtPARP2 genes are shown in Additional file 3: Figure S3D and E.

Because AtPARP3 is abundantly expressed in seeds but is poorly expressed in other tissues [30] (Additional file 3: Figure S3B and C; Additional file 4: Figure S4A and B), it would be easier to detect its activity in seeds if AtPARP3 had PARP enzymatic activity. AtPARP3 is presumed to play an important role in repairing DNA damage caused by seed dehydration or storage prior to the re-initiation of cell division during germination [30, 31]. As such, it might be an active PARP enzyme in seeds. However, in dry or germinating seeds, no PAR signal could be detected under normal conditions, neither after treatment with genotoxin. The RT-qPCR data also showed that AtPARP1/2/3 expressions in the early seed germinating stages (within $24 \mathrm{~h}$ ) were not responsive to zeocin or methyl methanesulfonate (MMS) treatment, which mainly induce double- and single-strand DNA breaks, respectively [41, 42] (Additional file 4: Figure $\mathrm{S} 4 \mathrm{C}$ and $\mathrm{D})$. Therefore, we modified our assay to a more robust one. Exogenous $\mathrm{NAD}^{+}$and broken DNA were supplemented in the protein extracts because $\mathrm{NAD}^{+}$is a known rate-limiting factor for PARP activity. In the presence of sufficient substrate $\left(\mathrm{NAD}^{+}\right)$and activating DNA, any residual PARP activity would be detectable. Surprisingly, we detected PAR formation in the seeds of wild-type, both parp 2 mutants and both parp 3 mutants, but not in the seeds of both parp1 mutants
(Fig. 6a). The PARylated proteins displayed as large smeared bands on the SDS-PAGE gel due to the sufficient provision of $\mathrm{NAD}^{+}$substrate, and the PAR chains were obviously long. The PAR signal strength detected in the two parp3 mutants was close to that of the wildtype, which indicated that AtPARP3 made no or an undetectable contribution to the PAR signal in seeds. In the parp1 parp2 ( $p 1 p 2)$ double mutant, no PAR signal was detected, which confirmed that AtPARP3 produced no PAR in vivo. Interestingly, in all mutants with the AtPARP1 mutation, such as the parp1, parp1 parp2, parp1 parp3 ( 1 1 3 ) and parp1 parp2 parp3 (p1 p2 p3) mutants, the PAR signal was undetectable, which indicated that even in seeds, AtPARP1 remains the main PARP responsible for PAR formation, although the expression level of AtPARP1 in seeds was much lower than that of AtPARP3 (Additional file 4: Figure S4A). To confirm the validity of the detected PAR signal in seeds, we added the competitive inhibitor $3-\mathrm{AB}$ to the reaction samples. 3-AB eliminated the signal almost completely (Fig. 6b), which indicated that the detected signal was a real PAR signal.

\section{AtPARP1 has predominant PARP enzymatic activity in response to zeocin and MMS treatments in seedlings}

The results obtained in seeds aroused our interest to investigate whether AtPARP1 also plays the predominant PAR formation role in seedlings. In humans, HsPARP1 contributes over $90 \%$ of the total PARP activity in vivo and is considered the key member of the PARP family [32, 43]. However, in Arabidopsis, AtPARP2, instead of AtPARP1, is reportedly the predominant PARP involved in both DNA damage and biotic stress responses in seedlings [20, 22]. We first examined the expression 


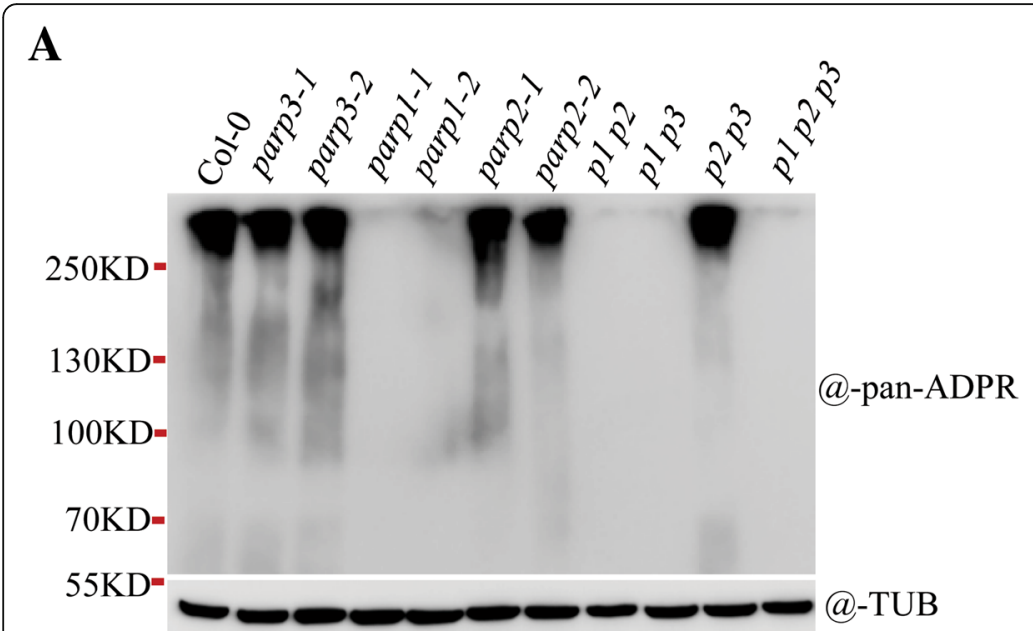

B

Fig. 6 AtPARP3 does not show poly (ADP-ribose) polymerase activity in seeds. a PAR signal generated in wild-type and mutant seeds in the presence of broken DNA and NAD . $\mathbf{b}$ The PARP inhibitor 3-AB can eliminate the PAR signal. Fifty milligrams of wild-type or various mutant seeds was incubated in distilled water for $24 \mathrm{~h}$. Total proteins were extracted from the seeds and then incubated with $1 \mathrm{mM} \mathrm{NAD}^{+}$in the presence of $500 \mathrm{nM}$ broken DNA for $10 \mathrm{~min}$ at $25^{\circ} \mathrm{C}$. PAR signals were detected using anti-pan-ADPR reagent. Anti-tubulin antibody was used in the immunoblot assay to show the protein loading amounts. p1 p2, parp1-3 parp2-3; p1 p3, parp1-1 parp3-1; p2 p3, parp2-1 parp3-1; p1 p2p3, parp1-3 parp2-3 parp3-1; and TUB, tubulin

levels of three PARP genes in Arabidopsis seedlings and found that AtPARP1 expression was highest while AtPARP3 expression was lowest under normal conditions (Additional file 4: Figure S4B). AtPARP3 could not be induced by zeocin and MMS in both seeds and seedlings (Additional file 4: Figure S4C, D, E, and F), whereas AtPARP1 and AtPARP2 transcriptions were strikingly induced by both zeocin and MMS in seedlings (Additional file 4: Figure S4E and F).

We then used the same parp mutants as those used in other laboratories to investigate PAR formation in response to the DNA-damaging agents zeocin and MMS. As expected, PAR was gradually induced in wild-type seedlings after treatment with genotoxin (Fig. 7a-d). All PAR signals originated from physiological PARP activity because the assay was performed without exogenous $\mathrm{NAD}^{+}$and activating DNA. Overall, the PAR signal generated under zeocin treatment was stronger than that under MMS treatment (Fig. 7a-d), which indicated that the stronger double-strand breakage agent zeocin induced more PAR formation than the single-strand break-inducing agent MMS. Longer exposure times and higher concentrations of genotoxins also generated stronger PAR signals. Interestingly, little or no PAR signal was observed in the parp1-1, parp1-2 and parp1-3 mutants, and neither did we detect the automodification activity of AtPARP2 in the three parp1 mutants within $24 \mathrm{~h}$ after genotoxin exposure (Fig. 7e and f), whereas AtPARP2 was well induced at these time points in wildtype seedlings (Fig. 7g). Weaker PAR signals were detected in the parp2-1, parp2-2 and parp2-3 mutants than in Col-0, which indicated that the mutation of
AtPARP2 decreased AtPARP1 activity. When grown on plates with MMS, all parp1 mutants displayed a mildly more sensitive phenotype than the parp2 mutants (Fig. 8c and e, please see Additional file 5: Table S1 for the source data); while on zeocin plates, although all seedlings could not grow up, the parp1 mutants had an averagely lower chlorophyll content than the parp $2 \mathrm{mu}$ tants (Fig. 8d and f). Taken together, our biochemistry and genetic data support the conclusion that AtPARP1, instead of AtPARP2, makes the greatest contribution to PAR formation in Arabidopsis and plays a dominant role in DNA damage response.

\section{In response to zeocin and MMS treatments, AtPARP1 is first activated, and then AtPARP2 is activated}

The time-dependent results of PAR formation in response to DNA-damaging agents (Fig. 7a-d) indicated that a blurred band first appeared around the size of AtPARP1, which is approximately $113 \mathrm{kD}$. This blurred band disappeared in all parp1 mutants (Fig. 7e and f), which suggested that it was correlated with AtPARP1. AtPARP1 activation upon genotoxin attack was consistent with the observation in humans that HsPARP1 is rapidly activated by genotoxin [44]. At approximately 48 $\mathrm{h}$ after treatment with $200 \mu \mathrm{g} / \mathrm{ml}$ zeocin and $72 \mathrm{~h}$ after treatment with $200 \mu \mathrm{g} / \mathrm{ml}$ MMS, a second band, which was the size of AtPARP2 (72 kD), started to appear (Fig. 7b and d), and this band disappeared in the parp2 mutant (Additional file 6: Figure S5), which indicated that it likely corresponds to AtPARP2. As shown in Fig. $7 \mathrm{~g}$, before $48 \mathrm{~h}$ AtPARP2 was already highly induced by zeocin (Fig. $7 \mathrm{~g}$ ), but no PAR signal was detected on 

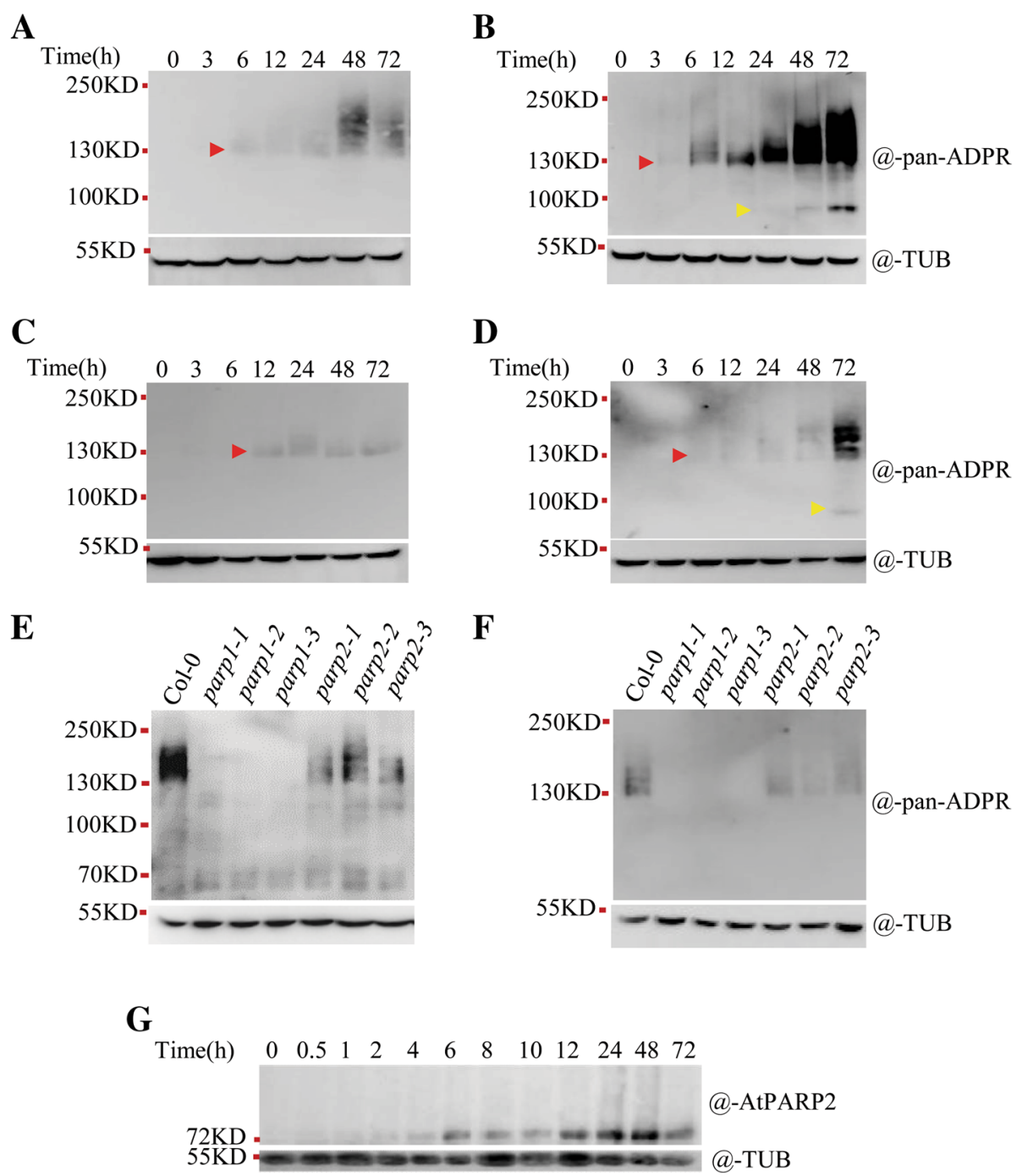

Fig. 7 AtPARP1 produces most of the PAR signal under zeocin and MMS treatments. a Time-dependent PAR signal generated by wild-type seedlings after zeocin treatment at $100 \mu \mathrm{g} / \mathrm{mL}$. b Time-dependent PAR signal generated by wild-type seedlings after zeocin treatment at $200 \mu \mathrm{g} /$ $\mathrm{mL}$. c Time-dependent PAR signal generated by wild-type seedlings after MMS treatment at $100 \mu \mathrm{g} / \mathrm{mL}$. d Time-dependent PAR signal generated by wild-type seedlings after MMS treatment at $200 \mu \mathrm{g} / \mathrm{mL}$. e PAR signal generated by different mutants of AtPARP1 and AtPARP2 after zeocin treatment at $100 \mu \mathrm{g} / \mathrm{mL}$ for $24 \mathrm{~h}$. f PAR signal generated by different mutants of AtPARP1 and AtPARP2 after MMS treatment at $200 \mu \mathrm{g} / \mathrm{mL}$ for 24 h. $\mathbf{g}$ Time-dependent induction of AtPARP2 by zeocin treatment. PAR signal was detected using anti-pan-ADPR reagent. AtPARP2 was detected with an anti-AtPARP2 antibody. Tubulin was detected using an anti-tubulin antibody to show the protein loading amounts. Red arrows indicate the poly (ADP-ribosyl) ated AtPARP1. Yellow arrows indicate the poly (ADP-ribosyl) ated AtPARP2. TUB, tubulin

AtPARP2, which implied that although AtPARP2 was produced, it might not undergo auto-PARylation at this moment.

To further test whether AtPARP2 was modified by AtPARP1 or by itself in vivo, we examined the changes of the AtPARP2 protein by immunoblot analysis. It was difficult to observe PARylation-induced band shift under physiological conditions when detected by anti-PARP2 antibody, likely due to the negative charges of PAR which weakened the binding of the antibody to the modified proteins, or due to too limited PARylation of AtPARP2 to be observed. To amplify the smear signal typical of PAR formation, we added $\mathrm{NAD}^{+}$to the samples; a very striking upward-blurred band was detected using anti-PARP2 antibody (Fig. 9a), and the upward traces were eliminated by $3-\mathrm{AB}$, which indicated that AtPARP2 was indeed poly (ADP-ribosyl) ated in the presence of exogenous $\mathrm{NAD}^{+}$. To assess whether the catalysis activity originated from AtPARP1 or AtPARP2 itself, we used the parp1, parp2, $p 1$ $p 2$ double mutant and parg1 mutant to observe the homeostasis of the PAR smears. The PAR signal was stronger in the parg1 mutant because PARG1 is mainly responsible for PAR hydrolysis in vivo, and the mutation of PARG1 allowed the easy detection of PARylated proteins (Fig. 9b). 
A

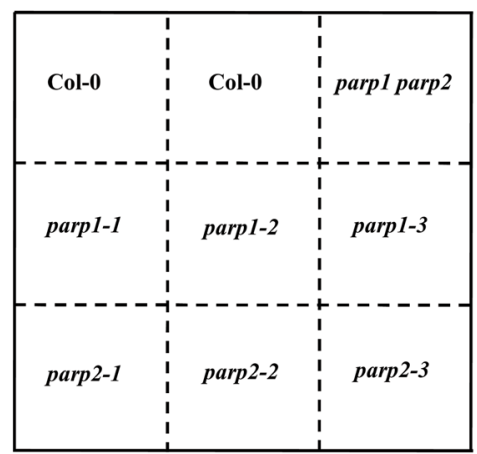

C

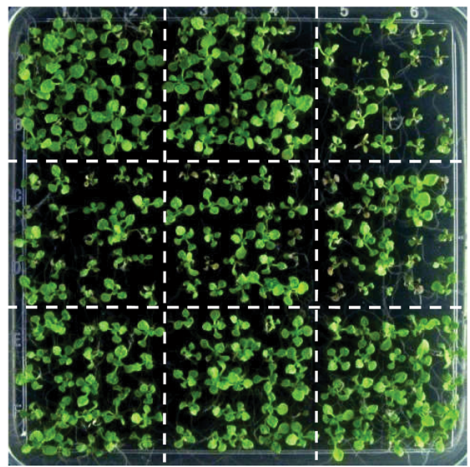

E

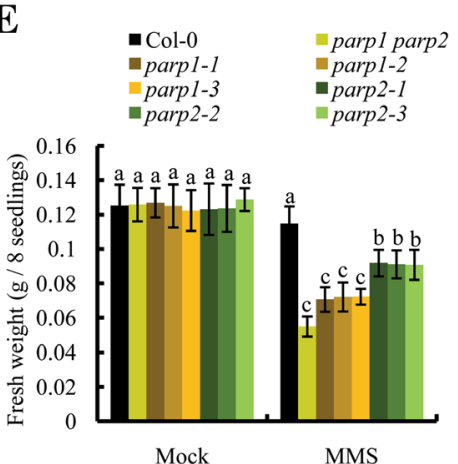

B

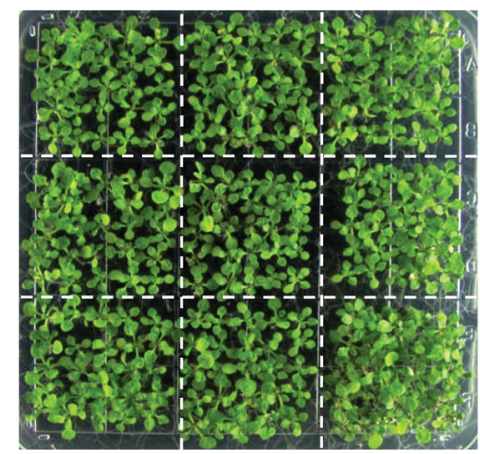

D

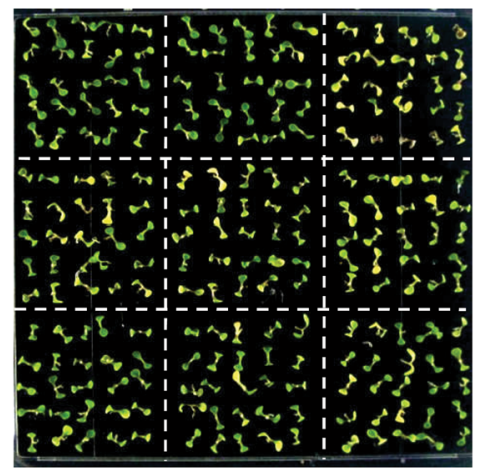

F

\begin{tabular}{|c|c|}
\hline - Col-0 & parp1 parp2 \\
\hline parp 1-1 & parp 1-2 \\
\hline parp 1-3 & - parp 2-1 \\
\hline parp $2-2$ & parp $2-3$ \\
\hline
\end{tabular}

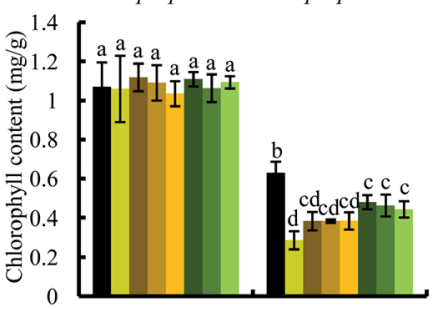

Mock

Zeocin

Fig. 8 The parp 1 mutants are more sensitive to MMS and zeocin treatments than the parp2 mutants. a Plants arrangement on the plates. $\mathbf{b}$ Phenotypes of Col-0 and mutants on 1/2 MS plates without treatment. c Phenotypes of Col-0 and mutants on 1/2 MS plates with 100 Mg/mL MMS. d Phenotypes of Col-0 and mutants on $1 / 2$ MS plates with $150 \mu \mathrm{g} / \mathrm{mL}$ zeocin. e Fresh weight of per eight seedlings with and without MMS treatment. $\mathbf{f}$ Chlorophyll content of seedlings with and without zeocin treatment. The seeds were sown on $1 / 2 \mathrm{MS}$ plates, stratified for $3 \mathrm{~d}$ at $4{ }^{\circ} \mathrm{C}$ in the dark, and then transferred into a growth chamber with $16 \mathrm{~h}$ of light/8 h of dark. Two-week-old seedlings were used for the assay. Each experiment was repeated at least three times. The results are shown as the means \pm SDs from three biological replicates. Two-way ANOVA with Bonferroni post hoc test analysis was performed. Different letters indicate significant differences $(P<0.05)$

In the parg1 mutant, we observed very obvious PARylated AtPARP2 at $48 \mathrm{~h}$ after zeocin treatment; however, the smearing PAR signal on AtPARP2 was absent in the parp1 mutant, which indicated that most of the PAR signal on AtPARP2 was generated by AtPARP1, and not by itself. AtPARP2 might not undergo or only undergo very limited auto-PARylation under this condition. This result, together with the previous results that under physiological conditions (without exogenous $\mathrm{NAD}^{+}$) the PARylation of AtPARP2 in parp1 mutants was not detected (Fig. 7 and
Additional file 6: Figure S5), suggested that under genotoxic stress, AtPARP1 is first activated and AtPARP2 is subsequently activated, and AtPARP2 is modified by AtPARP1 in vivo.

\section{Discussion}

Defining the biochemical activity of a protein is important for understanding its physiological function. Here, we demonstrated that AtPARP3 has lost PARP enzymatic activity due to the loss of $\mathrm{NAD}^{+}$-binding activity, 
A

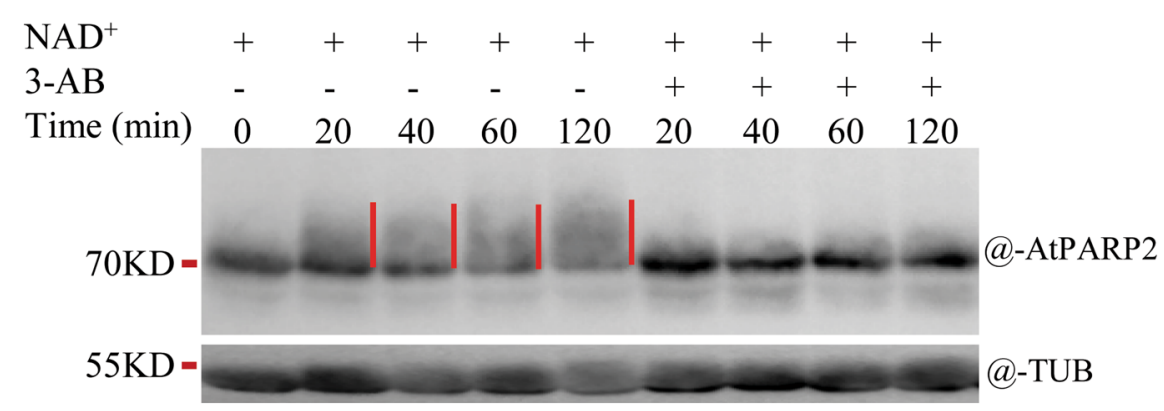

B

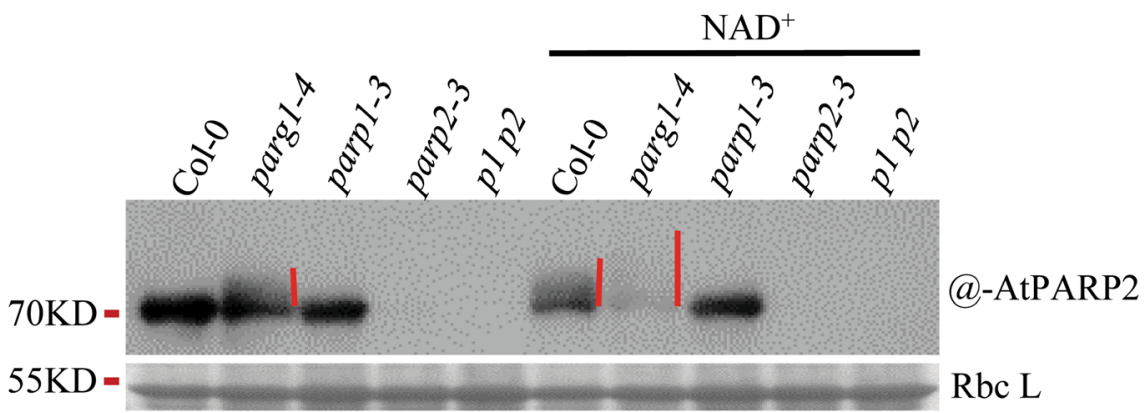

Fig. 9 AtPARP2 is poly (ADP-ribosyl) ated by AtPARP1 in vivo. a AtPARP2 is poly (ADP-ribosyl) ated in the presence of supplemented NAD ${ }^{+}$under zeocin treatment. $\mathbf{b}$ The poly (ADP-ribosyl) ation of AtPARP2 accumulated in the parg1 mutant and diminished in the parp1 mutant. The 10-d-old seedlings were treated with $200 \mu \mathrm{g} / \mathrm{mL}$ zeocin for $48 \mathrm{~h}$. The total proteins were extracted with or without exogenously supplemented $1 \mathrm{mM}$ $\mathrm{NAD}^{+}$, and PAR signals were detected using anti-pan-ADPR reagent. AtPARP2 was detected with an anti-AtPARP2 antibody. The immunoblot results with anti-tubulin antibody or the Coomassie blue-stained Rubisco large unit (Rbc $\mathrm{L}$ ) band were used to show the protein loading amounts. Red lines indicate the poly (ADP-ribosyl) ated AtPARP2. TUB, tubulin

although AtPARP3 is classified into the PARP family based on sequence homology. Therefore, other roles beyond PARP activity should be considered to determine its in vivo function. We also found that AtPARP1 acts as the predominant PARP in Arabidopsis in response to zeocin and MMS treatments, and AtPARP2 can be modified by AtPARP1 during the response to DNA damage.

Based on the phylogenetic analysis results (Additional file 2: Figure S2), most of the plant organisms used in this study have only one PARP3 member with the exception of Glycine max (two copies), which underwent a recent whole genome duplication event [45]. In addition, in the phylogenetic tree, plant PARP3 clustered close to human PARP1 rather than human PARP3, which suggests that PARP3 might have evolved to serve different functions in plants and animals. Plant PARP3 is mainly expressed in seeds. Because it has no PARP enzymatic activity, AtPARP3 likely plays a novel role, and this finding requires further research.

The crystal structure of human HsPARP1 provides important information for understanding the structure-activity relationship of PARP [33]. This analysis revealed that $\mathrm{N}$-terminal zinc fingers 1 and 3 (Zn1 and $\mathrm{Zn} 3$ ),
WGR domain, PRD/HD and the C-terminal catalytic domain (CAT) are the most important domains for PARP activity. Zn1, Zn3 and the WGR domain collectively bind to a double-strand break and cause distortion of the hydrophobic core of PRD/HD, which then reduces the thermal stability of the CAT domain and increases its dynamics [33].

The hydrophobic core of the PRD/HD domain is critical for the regulation of PARP activity. Mutagenesis of the interior leucine residues to hydrophilic amino acids can activate HsPARP1 in the absence of DNA [33], mimicking the DNA damage-induced PRD/HD distortion. We found that a few conserved leucine residues in the PRD/HD domain (from S634 to H768) of AtPARP1 have been replaced by hydrophilic amino acids in the PRD/HD domain of AtPARP3 (from A449 to Y587), which might explain why placement of the AtPARP1 catalytic domain in AtPARP3 (AtP3-P1) results in a constitutively active protein without activating DNA (Fig. 2b). A more hydrophilic core of the PRD/HD domain of AtPARP3 relative to AtPARP1 likely distorted the PRD/HD domain and activated the AtPARP1 catalytic domain in the AtP3-P1 chimera protein. We may create a novel constitutively active PARP protein without the need for DNA activation by utilizing the PRD/HD 
domain of AtPARP3, which has potential value for studying the functions of PARPs.

Among the 17 members of the human PARP family, only HsPARP1, HsPARP2 and HsPARP3 are known to be involved in DNA repair [2, 32]. These members are evolutionarily closer to each other than to other members. Arabidopsis PARP1 and PARP2 correspond to human PARP1 and PARP2, respectively, based on both sequence homology and domain architecture (Additional file 1: Figure S1) [18, 19]. However, Arabidopsis PARP2 was previously reported to play the predominant role in both the DNA damage and biotic stress responses [20, 22], and AtPARP2 was shown to have stronger in vitro activity than AtPARP1.

Obviously, our results do not support this conclusion, potentially due to the different experimental conditions used in different studies. Firstly, it is unknown whether the MABE1016 reagent and antiPAR antibodies have different sensitivities and specificities for different types of PAR. The anti-panADP-ribose binding reagent (MABE1016, Merck), claimed to be able to detect both mono (ADP-ribose) and poly (ADP-ribose), was substantially more sensitive than the anti-poly (ADP-ribose) polymer antibody [10H] (ab14459, Abcam) in our experiments for PAR detection, particularly for the detection of PAR in plant tissues. No signals appeared on the blot when detected by anti-PAR antibody, but strong PAR signals could be observed by anti-panADP-ribose binding reagent using the same samples (Additional file 7: Figure S6A and B). Anti-PAR antibody could only detect the PAR signals when exogenous $\mathrm{NAD}^{+}$and activated DNA were added into the extraction buffer to enhance PARP catalysis reaction (Additional file 7: Figure $\mathrm{S6C}$ ), but this is not a physiological condition since $\mathrm{NAD}^{+}$amount is limited in vivo. Under this condition, all potential substrates might be PARylated by PARPs. Even so, most of the PAR signals disappeared in three parp1 mutants, again supporting that AtPARP1 contributes most of the PARP activity.

Secondly, for the in vitro experiments, different protein expression systems were used. Both AtPARP1 and AtPARP3 have 21 cysteine acids, whereas AtPARP2 has only six cysteine acids. Disulfide formation might not be a problem for AtPARP2 but could induce abnormal folding of AtPARP1 in E. coli. Therefore, selection of the proper expression vector for AtPARP1 is particularly important. We used the pET32a $(+)$ vector to produce AtPARPs because the thioredoxin tag in this vector can facilitate the correct disulfide bonding of foreign proteins in host strains such as BL21(DE3)trxB ${ }^{-}$and Origami (DE3), as detailed in the manufacturer's manual. The recombinant protein TRXH-AtPARP1 showed robust activity in our assays, producing large amounts of PAR within seconds, which is comparable to human PARP1. A maltose-binding domain (MBD) tag was used in previous studies to express recombinant AtPARP1 and AtPARP2 proteins, and in our side-by-side experiments, the MBD-fused AtPARP1 and AtPARP2 were also less active than the TRXH-fused AtPARP1 and AtPARP2, respectively (Additional file 8: Figure S7A and B), and in general, AtPARP2 produced shorter PAR chains than AtPARP1 within the same incubation time periods in our assay.

Finally, for the in vivo assay, different genotoxins have been used to induce DNA breaks. Bleomycin and mitomycin (MMC) treatments were used in a previous study [22], whereas zeocin and MMS were used in our experiments. Although zeocin belongs to the bleomycin family, it exerts milder DNA-damaging effects than bleomycin (product data sheets available at www.calbiochem.com). In our side-by-side experiments with bleomycin and zeocin, we constantly observed that AtPARP1 was responsible for the majority of PAR production (Additional file 9: Figure S8B and $\mathrm{C}$ ). Under milder genome stimulus, such as treatments with low or medium doses of double- or single-strand DNA-breaking agents, only the activation of AtPARP1 was observed within 2 days (Fig. $7 \mathrm{a}$ and c), and after that, AtPARP2 was mainly PARylated by AtPARP1 (Fig. 7, Fig. 9 and Additional file 6: Figure S5). Even so, the possibility that AtPARP2 modifies itself cannot be excluded at the later stages of the DNA damage response or under fiercer DNA-damaging conditions.

Our phenotypic analysis also showed that all three parp1 mutants were more sensitive to genotoxic stress than the three parp2 mutants (Fig. 8), supporting an important role of AtPARP1 in the Arabidopsis DNA damage response. In animals, AtPARP1 binds to DNA damage sites in less than $1 \mathrm{~s}$ [46], and thus, a role in rapid DNA damage detection is assigned to this protein. AtPARP1 might also act as a rapid detector of DNA breaks in plant cells. By interacting with AtPARP2 [22], it modifies AtPARP2 after its self-activation, and then AtPARP1 and AtPARP2 coordinately participate in the DNA repair process. The phylogenetic relationship and the structural similarity strongly indicate that Arabidopsis PARP1 is closer to animal PARP1, which contributes more than $90 \%$ of PARP activity in vivo [43]. Our biochemical and genetic data also support the notion that AtPARP1, similar to HsPARP1 [46, 47], plays a leading role in the DNA damage response in Arabidopsis.

\section{Conclusions}

Taken together, our results demonstrated that AtPARP3 does not act as a PARP enzyme in seeds, which implied that other roles beyond PARP enzymatic activity need to be considered for AtPARP3. In 
addition, AtPARP1, rather than AtPARP2 and AtPARP3, is the major enzyme responsible for PARylation in both seeds and seedlings in response to zeocin and MMS treatments, supporting a conserved predominant role for PARP1 in the DNA damage response in both animals and plants.

\section{Methods}

\section{Plant materials and growth conditions}

All Arabidopsis seeds were of the Col-0 ecotype background. Sequence data of the genes studied in this article can be found on the TAIR website (https://www. arabidopsis.org/) or GenBank database under the following accession numbers: AtPARP1 (At2g31320), AtPARP2 (At4g02390), AtPARP3 (At5g22470), and AtPARG1 (At2g31870). The Col-0 and T-DNA insertion line seeds parp3-1 (Salk_108092C), parp3-2 (Sail_632_D07) and parg1-4 (Salk_012110) were ordered from the TAIR database. Other T-DNA mutants were kindly provided by the following laboratories: parp1-1, parp1-2, parp21, parp2-2, parp1 parp3, and parp2 parp3 seeds were obtained from Dr. Edgar Peiter's Lab at Martin Luther University Halle-Wittenberg, and parp1-3, parp2-3, and parp 1 parp 2 seeds were obtained from Dr. de Pater's $\mathrm{Lab}$ at Leiden University. These lines can be found under the following stock numbers in the TAIR database: parp1-1 (GK_380E06), parp1-2 (GK_382F01), parp1-3 (GK_692A05), parp2-1 (GK_420G03), parp2-2 (Sail_1250_B03), and parp2-3 (Salk_140400).

Homozygous individuals were identified by genomic DNA PCR, RT-qPCR or immunoblotting analysis. The PCR primers used for identification of the mutants are listed in Additional file 10: Table S2. The mutants parg1-4, parp1-1, parp1-2, parp1-3, parp2-1, parp2-2, parp2-3, and $p 1$ p2 (parp1-3 parp2-3) were described previously $[23,24,29,30] . p 2 p 3$ was constructed using parp2-3 and parp3-1; $p 1$ p3 was obtained by crossing parp 1-3 and parp3-1; and $p 1$ p2 p3 was obtained using parp1-3, parp2-3 and parp3-1. The seedlings were grown in a growth chamber under a photoperiodic cycle consisting of 16 $\mathrm{h}$ of light and $8 \mathrm{~h}$ of dark at $22^{\circ} \mathrm{C}$.

\section{RNA extraction and RT-qPCR}

Total RNA from Arabidopsis seedlings was extracted using the TRIzol reagent (TaKaRa, Japan), and the total RNA from seeds was extracted as described previously [48]. RNA was reverse-transcribed with the PrimeScript ${ }^{\mathrm{m}}$ RT reagent kit with gDNA Eraser (TaKaRa, Japan). qPCR was performed with the SYBR Premix Ex Taq ${ }^{\text {tm }}$ kit (TaKaRa, Japan) using the CFX96 $6^{\text {TM }}$ Real-Time PCR Detection System (Bio-Rad, USA). The Arabidopsis UBQ5 gene was used as an internal control to normalize the different samples. The RT-qPCR primers are listed in
Additional file 10: Table S2. Three biological replicates were analyzed for each sample.

\section{Protein expression and ADP-ribosylation assay}

The coding sequences of AtPARP1 and AtPARP2 were amplified from the cDNA of 10-d-old seedlings. The AtPARP1 coding region was cloned into the pET32a $(+)$ vector with the TRXH tag and PMAL-c5G vector with the maltose-binding domain (MBD) tag using Sac I/Not I and Nde I/Sal I sites, respectively, and AtPARP2 was cloned into pET32a(+) using Bam HI/Xho I sites. The MBP-AtPARP2 plasmid was provided by Dr. He Ping [20]. The AtPARP3 coding sequence was amplified from Arabidopsis dry seeds and cloned into the pET32a(+) vector and pGEX-4 T-1 vector with a GST tag using Sal I/Not I sites. The protein expression constructs were transformed into Origami (DE3) competent cells based on the standard protocol (Novagen, Germany). The empty vectors were also transformed for tag protein expression. The tag proteins served as negative controls in the biochemical activity assay. Recombinant proteins were purified with a column filled with Nickle Sepharose Fast Flow (GE Healthcare, Sweden). The purified proteins were dialyzed completely against $10 \mathrm{mM}$ Tris- $\mathrm{HCl}$ (pH 7.5) buffer to remove salts. The proteins $(1-10 \mu \mathrm{g})$ were aliquoted into each tube containing $20 \mathrm{mM}$ Tris- $\mathrm{HCl}$ (pH 7.5), $50 \mathrm{mM} \mathrm{NaCl}, 7.5 \mathrm{mM} \mathrm{MgCl}_{2}, 0.2 \mathrm{mM}$ DTT, and $500 \mathrm{nM}$ synthesized DNA oligos [35]. To initiate the reaction, $\mathrm{NAD}^{+}$(Sigma Aldrich, USA) was added to each tube to a final concentration of $1 \mathrm{mM}$. The reactions were continued at room temperature for the desired time periods and then terminated by adding a 3-fold volume of prechilled acetone. After centrifugation, the precipitated proteins were subjected to SDS-PAGE for immunoblotting assays or Coomassie blue staining. The immunoblots were probed with anti-pan-ADP-ribose binding reagent (Merck, Germany), anti-His antibody (Proteintech, USA), antiAtPARP1 polyclonal antibody (against the E.coli-expressed full-length AtPARP1) (Youke Biotech, China) and antiAtPARP3 polyclonal antibody (against the E.coli-expressed AtPARP3 fragment 160S-392K) (Youke Biotech, China), respectively for detecting different signals. After chemiluminescence reaction, the protein bands were visualized using a ChemScope 3300 mini instrument (CLINX, China).

\section{Dot blot}

The purified proteins were blotted onto the methanol-activated PVDF membrane (Millipore, USA). After drying at room temperature, the membranes were incubated with $25 \mu \mathrm{M}$ biotinylated $\mathrm{NAD}^{+}$(Trevigen, USA) and $500 \mathrm{nM}$ synthesized DNA oligos for $30 \mathrm{~min}$ at $25^{\circ} \mathrm{C}$. The blot was washed with TBST buffer $(50 \mathrm{mM}$ Tris, $0.5 \mathrm{M} \mathrm{NaCl}$, and $0.05 \%$ Tween-20, $\mathrm{pH} 8.0$ ) and then incubated with streptavidin/HRP (Solarbio, China) at room temperature for $2 \mathrm{~h}$. After reaction with chemiluminescent substrates, 
fluorescence signals were detected using a ChemScope 3300 mini instrument (CLINX, China). BSA and tag proteins were used as negative controls, and AtPARP1 was used as the positive control. The signals obtained with antiHis antibody (Proteintech, USA) were used as the protein loading control for each spot.

\section{Protein extraction and immunoblotting}

To detect the PAR signal in seedlings, 10-d-old seedlings were treated with different concentrations of zeocin or MMS for $48 \mathrm{~h}$. The seedlings $(100 \mathrm{mg})$ were homogenized in liquid nitrogen and then suspended in protein extraction buffer $(50 \mathrm{mM}$ Tris- $\mathrm{HCl}$ pH 8.0, $100 \mathrm{mM}$ $\mathrm{NaCl}, 5 \mathrm{mM} \mathrm{MgCl} 2,10 \%$ glycerol, $0.1 \% \mathrm{NP}-40$ and protease inhibitor cocktail). The supernatant was isolated by centrifugation, and after SDS loading buffer was added to the sample, the sample tube was boiled for $10 \mathrm{~min}$. After SDS-PAGE analysis, the proteins were blotted onto Immobilon-P Transfer membranes (Millipore, USA). The PAR levels were detected using anti-Pan-ADPR binding reagent or anti-poly (ADP-ribose) polymer antibody $[10 \mathrm{H}]$ (ab14459, Abcam, UK). AtPARP2 was detected using an anti-AtPARP2 polyclonal antibody (against the E.coli-expressed full-length AtPARP2) (Youke Biotech, China), and tubulin was detected with anti-tubulin antibody (Beyotime, China) as a loading control. The protein signals were visualized using a ChemScope 3300 mini instrument (CLINX, China).

To detect the PAR signal in seeds, dry seeds $(50 \mathrm{mg})$ were imbibed in distilled water for $24 \mathrm{~h}$, and the total proteins were extracted using protein extraction buffer. After carefully removing the surface-floating insoluble and fatty acid components, the supernatant proteins were incubated for $10 \mathrm{~min}$ at $25^{\circ} \mathrm{C}$ with $1 \mathrm{mM} \mathrm{NAD}^{+}$ and $500 \mathrm{nM}$ DNA oligos. The immunoblotting was performed as described above.

\section{Molecular docking and binding affinity calculation}

The protein structures of HsPARP1, HsPARP2, HsPARP3, HsPARP5a and HsPARP5b have been resolved, and the structural data can be downloaded from the RCSB protein data bank (PDB, https://www.rcsb.org/). No crystal structures are available for AtPARP1, AtPARP2 and AtPARP3; therefore, we used the online structure prediction software Phyre2 [38] (http://www.sbg.bio.ic.ac.uk/phyre2/html/ page.cgi?id=index) to predict their structures. The generated structures of AtPARP1, AtPARP2 and AtPARP3 together with the resolved structures of HsPARP1, HsPARP2, HsPARP3, HsPARP5a and HsPARP5b were used for molecular docking.

The structure file for $\mathrm{NAD}^{+}$was generated using ChemBio3D software. The open source program AutoDock Vina [39] was used to perform the molecular docking experiment. Specifically, the protein was set as a rigid structure, and the $\mathrm{NAD}^{+}$molecule was flexible, with all of the rotatable bonds (17 out of 32) free to rotate. The coordinates of the alpha carbon in histidine (cysteine in AtPARP3) were used to center the docking grid box because the histidine and tyrosine residues of the triad motif are known to be involved in $\mathrm{NAD}^{+}$binding. The size of the docking grid box was 22.5*22.5*22.5 (in Angstrom units) for all proteins.

For each protein, the molecular docking experiment was repeated five times with the same parameters, and nine binding modes with different binding affinities were generated each time. The mean of the top binding affinity from the various time points was used to evaluate the protein's binding affinity to the $\mathrm{NAD}^{+}$molecule.

\section{Sequence and motif analyses}

Alignments of the PARP protein sequences were performed using the MAFFT server (https://www.ebi.ac.uk/ Tools/msa/mafft/) [49]. The conserved functional domains of PARPs were predicted with the NCBI CDD tool (www.ncbi.nlm.nih.gov/cdd) using the structural information provided by the TAIR server (www.Arabidopsis.org) as a reference.

\section{Identification of PARP1/2/3 subfamily members and phylogenetic analysis}

Twenty-eight representative species were selected for the phylogenetic analysis of the PARP $1 / 2 / 3$ subfamily in the PARP gene family (Additional file 11: Table S3). The genomic and proteomic sequences of 19 selected species (Amborella trichopoda, Arabidopsis thaliana, Brassica rapa, Capsella rubella, Carica papaya, Cucumis sativus, Glycine max, Medicago truncatula, Mimulus guttatus, Oryza sativa, Phaseolus vulgaris, Physcomitrella patens, Populus trichocarpa, Theobroma cacao, Selaginella moellendorffii, Sorghum bicolor, Solanum tuberosum, Vitis vinifera and Zostera marina) were downloaded from Phytozome version 12 [50]. The proteomic sequences of Nelumbo nucifera, Picea abies and Ginkgo biloba were downloaded from https://omictools.com/lotus-db-tool [51], ConGenIE [52] and GigaDB [53], respectively. The proteomic sequences of four animal (Caenorhabditis elegans, Drosophila melanogaster, Homo sapiens, and Mimulus guttatus) and two fungal (Mycena alexandri and Sarcoscypha coccinea) species were retrieved from Ensembl [54] and MycoCosm [55], respectively.

To identify the members of the PARP $1 / 2 / 3$ subfamily, the protein sequences of AtPARP1, 2 and 3 were used as queries to blast against the proteomes of the 28 selected species using DIAMOND blastp [56] with E-value $\leq 1 \times 10^{-5}$. According to the Pfam analysis [57], the target dataset was screened to remove sequences without a WGR, PARP_reg or PARP domain. Only homologs containing all three domains 
with identity $>50$ were selected as PARP $1 / 2 / 3$ subfamily proteins in this study. Multiple protein sequences were aligned using MAFFT [49] with the default parameters, manually adjusted using AliView [58], and then trimmed using trimAL [59] with the option "-gt 0.6". To illustrate the evolutionary relationships between the PARP $1 / 2 / 3$ subfamilies, the maximum likelihood (ML) phylogenetic tree was constructed using RAxML version 8.1.17 [60] with the "PROTCATJTT" model. The bootstrap significance test was performed with 100 replicates.

\section{Chlorophyll extraction}

Approximately $20 \mathrm{mg}$ of total seedlings was homogenized in liquid nitrogen and then extracted in $95 \%$ acetone for $15 \mathrm{~min}$ in the dark. The supernatant solution was isolated by centrifugation for $10 \mathrm{~min}$ and used to determine the OD645 and OD663 values through a spectrophotometric analysis. The total chlorophyll content was calculated according to the following formula: $\mathrm{Chl}$ $\mathrm{a}+\mathrm{Chl} \mathrm{b}=(20.21 *$ OD645 + 8.02* OD663) / $1000 *$ volume / weight.

\section{Domain swapping and point mutagenesis of PARP proteins}

The recombinant protein AtP1-P3 includes amino acids 1-628 of AtPARP1 and amino acids 424-814 of AtPARP3. The AtP3-P1 protein consists of amino acids 1-423 of AtPARP3 and amino acids 629-984 of AtPARP1. The cDNA fragments of these regions were ligated using a NovoRec ${ }^{\circ}$ PCR one-step clone kit (SinoBio, China). For point mutations, the catalytic triad of AtPARP1 is composed of three amino acids, H833Y867-E960, whereas that of AtPARP3 consists of the C653-V687-E782 amino acids. The coding sequences of proteins with the catalytic triad point mutations were obtained by a two-step PCR site-directed method [61]. In detail, the DNA sequence of AtPARP1 or AtPARP3 was divided into two fragments. The first point mutation was designed in one pair of primers, and PCR was performed to amplify the first fragment. The second point mutation was then included in another pair of primers and accomplished using the same method. The two PCR fragments were ligated by PCR using the full-length primers. The full-length fragment with two point mutations was cloned into the pET32a(+) vector using Sal I/ Not I sites. All the primers used in the above experiments are listed in Additional file 10: Table S2.

\section{Additional files}

Additional file 1: Figure S1 Domain architecture and sequence analysis of PARP members in Arabidopsis and humans. (A) Comparison of the domain architecture between human and Arabidopsis PARP1, PARP2 and PARP3. (B) Motif-based sequence alignment of the PARP signature of Arabidopsis PARP1, PARP2 and PARP3. Stars show the conserved H-Y-E triad in AtPARP1 and AtPARP2, which has an alternate form in AtPARP3. Red frames show the sequence motifs 1,2 and 3. The letters with the same color represent conserved amino acids. (PDF $10681 \mathrm{~kb}$ )

Additional file 2: Figure S2 Maximum likelihood (ML) phylogenetic tree of the PARP $1 / 2 / 3$ subfamily members in 28 representative species. The Arabidopsis thaliana PARP3 protein is marked by a red solid triangle. Orange solid asterisks on nodes denote gene duplication events. The bootstrap values (>50) with 100 replicates are given for each node on the tree. Genes from plants, animals and fungi are given in Additional file 11: Table S3. (PDF $8823 \mathrm{~kb}$ )

Additional file 3: Figure S3 Mutants of Arabidopsis PARP genes used in this study. (A) T-DNA insertion sites in Arabidopsis parp3 mutants. parp31, Salk_108092C; parp3-2, Sail_632_D07. Exons are represented by filled boxes, introns are represented by dark lines, and T-DNA insertions are indicated by filled triangles. (B) RT-qPCR analysis of the AtPARP3 expression levels in Col-0 and parp3 mutant seeds. Dry seeds were used for RT-qPCR. The AtUBQ5 gene was used as the internal control. (C) Detection of AtPARP3 protein in seeds with anti-AtPARP3 antibody. Fifty milligrams of dry seeds was used for the extraction of total protein from each sample. The blotting results with anti-tubulin antibody served as loading controls. (D) T-DNA insertion sites in parp1 mutants. parp1-1, GK_380E06; parp1-2, GK_382F01; and parp1-3, GK_692A05. (E) T-DNA insertion sites in parp2 mutants. parp2-1, GK_420G03; parp2-2, Sail_1250_B03; and parp2-3, Salk_140400. The results are shown as the means \pm SDs from three biological replicates. TUB, tubulin. (PDF $4047 \mathrm{~kb}$ )

Additional file 4: Figure S4 Transcription levels of AtPARPs under normal conditions and genotoxin treatments in seeds and seedlings. (A) Relative expression levels of the AtPARP members in Arabidopsis dry seeds. (B) Expression levels of the AtPARP members in Arabidopsis seedlings after distilled water treatment. (C) Comparison of the expression levels of the AtPARP members in seeds after MMS treatment. (D) Comparison of the expression levels of the AtPARP members in seeds after zeocin treatment. (E) Comparison of the expression levels of the AtPARP members in Arabidopsis seedlings after MMS treatment. (F) Comparison of the expression level of the PARP members in Arabidopsis seedlings after zeocin treatment. Seeds of Col-0 and parp mutants were incubated with distilled water (A), $100 \mu \mathrm{g} / \mathrm{mL}$ MMS (C), or $200 \mu \mathrm{g} / \mathrm{mL}$ zeocin (D) for different time periods. 10-d-old Col-0 and parp mutants seedlings grown on $1 / 2$ MS plates were sprayed with distilled water (B), $100 \mu \mathrm{g} / \mathrm{mL}$ MMS (E), or $200 \mu \mathrm{g} / \mathrm{mL}$ zeocin (F) for different time periods. Total RNA was extracted from seeds or seedlings and subjected to RTqPCR analysis. The expression levels of AtPARPs were normalized to that of AtUBQ5. Simultaneous mock experiments were performed by treating plants with distilled water, and fold changes were calculated by normalizing the gene expression levels under treatment to that of the corresponding mock experiment. The results are shown as the means \pm SDs from three biological replicates. (PDF $12025 \mathrm{~kb}$ )

Additional file 5: Table S1 Source data of seedling fresh weight and chlorophyll content under genotoxin treatment. (XLSX $32 \mathrm{~kb}$ )

Additional file 6: Figure S5 Comparison of PAR signals in Col-0, parp1 and parp2 mutants. 10-d-old seedlings were treated by $200 \mu \mathrm{g} / \mathrm{mL}$ zeocin for $48 \mathrm{~h}$. The total proteins in the seedlings were extracted, blotted and then detected using anti-pan-ADPR reagent. Tubulin was detected using an anti-tubulin antibody to show the protein loading amounts. TUB, tubulin. (PDF $2355 \mathrm{~kb}$ )

Additional file 7: Figure $\mathbf{S 6}$ Comparison of the PAR signals detected by anti-pan-ADPR reagent and anti-PAR antibody, respectively. (A) PAR signals in different plants were detected by anti-pan-ADP-ribose binding reagent. (B) PAR signals in different plants were detected by anti-PAR antibody. No signal could be detected on the membrane. (C) PAR signals in different plants were detected by anti-PAR antibody with exogenous $\mathrm{NAD}^{+}$and activated DNA in the extraction buffer. $0.3 \mathrm{mM} \mathrm{NAD}{ }^{+}$and 100 $\mathrm{nM}$ broken DNA were added into the protein extraction buffer to enhance the PARP catalysis reactions. For (A), (B) and (C), 10-d-old seedlings were treated by $200 \mu \mathrm{g} / \mathrm{mL}$ zeocin for $48 \mathrm{~h}$ and the total proteins in the seedlings were extracted and used for western blot. For 
(B), the samples are the same as those in (A) but detected with anti-PAR antibody. (C), Total proteins were extracted using the same buffer as (A) and (B) except in it $0.3 \mathrm{mM} \mathrm{NAD}$ and $100 \mathrm{nM}$ broken DNA were added. TUB, tubulin; @-pan-ADPR, anti-pan-ADPR reagent; @-PAR, anti-PAR antibody. (PDF $7691 \mathrm{~kb}$ )

Additional file 8: Figure S7 Comparison of the activities of MBD-fused and TRXH-fused recombinant AtPARP proteins. (A) Comparison of the activities of different tag-fused AtPARP1 proteins. (B) Comparison of the activities of different tag-fused AtPARP2 proteins. The purified proteins were incubated with $500 \mathrm{nM}$ DNA and $1 \mathrm{mM} \mathrm{NAD}^{+}$at $25^{\circ} \mathrm{C}$ for different time periods. After the reaction, the proteins were analyzed by immunoblotting with anti-pan-ADPR reagent (the upper panel). Arrows in the bottom Coomassie blue-stained gel indicate the recombinant proteins TRXH-AtPARP1 (red), MBD-AtPARP1 (black), TRXH-AtPARP2 (cyan), and MBD-AtPARP2 (blue). (PDF $7207 \mathrm{~kb}$ )

Additional file 9: Figure S8 AtPARP1 was responsible for the generation of most of the PAR signals under bleomycin and zeocin treatments. (A) PAR signals in different parp1 and parp2 mutants after mock $\left(\mathrm{H}_{2} \mathrm{O}\right)$ treatment for $24 \mathrm{~h}$ and $48 \mathrm{~h}$, respectively. (B) PAR signals in different parp1 and parp2 mutants after $200 \mu \mathrm{g} / \mathrm{mL}$ zeocin or $25 \mu \mathrm{g} / \mathrm{mL}$ bleomycin treatment for $24 \mathrm{~h}$. (C) PAR signals in different parp1 and parp2 mutants after $200 \mu \mathrm{g} / \mathrm{mL}$ zeocin or $25 \mu \mathrm{g} / \mathrm{mL}$ bleomycin treatment for $48 \mathrm{~h}$. 10-d-old seedlings were treated with zeocin or bleomycin for different time periods and then the total proteins were extracted and detected using anti-pan-ADPR reagent. Tubulin was detected using an anti-tubulin antibody to indicate the protein loading amounts. TUB, tubulin; @-pan-ADPR, anti-pan-ADPR reagent. (PDF 9141 kb)

Additional file 10: Table S2 Primers used in this work. (PDF $12294 \mathrm{~kb}$ ) Additional file 11: Table S3 List of genes used for phylogenetic analysis of the PARP $1 / 2 / 3$ subfamily in the PARP family. (PDF $10506 \mathrm{~kb}$ )

\section{Abbreviations}

3-AB: 3-aminobenzamide; BRCT: BRCA-1 C-terminus; BSA: albumin from bovine serum; CAT: C-terminal catalytic domain; C-G-S: cysteine-glycine-serine: C-V-E: histidine-valine-glutamic acid; GST: glutathione S-transferase; HD: Helical subdomain; H-G-S: histine-glycine-serine; H-Y-E: histidine-tyrosine-glutamic acid; MAMP: microbe- associated molecular pattern; mART: mono (ADP-ribosyl)ation; MBD: maltose-binding domain; MMC: mitomycin; MMS: methyl methanesulfonate; PAR: poly (ADP-ribose); PARG: poly (ADP-ribose) glycohydrolase; PARP: poly (ADP-ribose) polymerase; PARylation: poly (ADP- ribosyl)ation; PRD: PARP regulatory domain; PVDF: polyvinylidene fluoride; SAP: SAF/Acinus/PIAS motif; TRXH: thioredoxin and histidine; V-C-S: valine-phenylalanine-alanine; WGR: Trp-Gly-Arg; Y-F-A: tyrosinephenylalanine-alanine

\section{Acknowledgements}

We thank Dr. Ping He at Texas A\&M University for kindly providing the MBP-PARP2 expression vectors, Dr. Edgar Peiter at Martin Luther University Halle-Wittenberg for providing the parp1-1, parp1-2, parp2-1, parp2-2, parp1 parp3, and parp2 parp3 seeds, and Dr. de Pater at Leiden University for providing the parp1-3, parp2-3, and parp1 parp2 seeds.

\section{Authors' contributions}

XG conceived the study and designed the experiments. ZG, WP, WC, QL, QW, XC and ZL performed the experiments. XG and ZG wrote the manuscript. All the authors have read and approved the final manuscript.

\section{Funding}

This work was supported by the National Natural Science Foundation of China (Grants No. 31770274 and 31170169) and the Shanghai Science and Technology Innovation Action Plan 18JC1411800. These funders were not involved in the design of the study, data collection and analysis, or writing of the manuscript

\section{Availability of data and materials}

The datasets supporting the conclusions described in this article are included within the manuscript and its additional files, and the raw data are available from the corresponding author upon reasonable request.

\section{Ethics approval and consent to participate}

Not applicable.

\section{Consent for publication}

Not applicable.

\section{Competing interests}

The authors declare that they have no competing interests.

Received: 31 January 2019 Accepted: 31 July 2019

Published online: 19 August 2019

\section{References}

1. Kim MY, Zhang T, Kraus WL. Poly (ADP-ribosyl) ation by PARP-1: 'PAR-laying' NAD+ into a nuclear signal. Genes Dev. 2005;19(17):1951-67.

2. Gibson BA, Kraus WL. New insights into the molecular and cellular functions of poly (ADP-ribose) and PARPs. Nat Rev Mol Cell Biol. 2012;13(7):411-24.

3. Luo $X$, Kraus WL. On PAR with PARP: cellular stress signaling through poly (ADP-ribose) and PARP-1. Genes Dev. 2012;26(5):417-32.

4. Feng B, Ma S, Chen S, Zhu N, Zhang S, Yu B, Yu Y, Le B, Chen X, Dinesh-Kumar $\mathrm{SP}$, et al. PARylation of the forkhead-associated domain protein DAWDLE regulates plant immunity. EMBO Rep. 2016;17(12):1799-813.

5. Krishnakumar R, Kraus WL. The PARP side of the nucleus: molecular actions, physiological outcomes, and clinical targets. Mol Cell. 2010;39(1):8-24.

6. Coyne GOS, Chen A, Kummar S. Delivering on the promise: poly ADP ribose polymerase inhibition as targeted anticancer therapy. Curr Opin Oncol. 2015;27(6):475-81

7. Anders CK, Winer EP, Ford JM, Dent R, Silver DP, Sledge GW, Carey LA. Poly (ADP-ribose) polymerase inhibition: "targeted" therapy for triple-negative breast cancer. Clin Cancer Res. 2010;16(19):4702-10.

8. Phillips R, Hawkins SW. Characteristics of the inhibition of induced tracheary element differentiation by 3-aminobenzamide and related compounds. J Exp Bot. 1985;36(1):119-28

9. Schulz P, Neukermans J, van der Kelen K, Muhlenbock P, van Breusegem F, Noctor G, Teige M, Metzlaff M, Hannah MA. Chemical PARP inhibition enhances growth of Arabidopsis and reduces anthocyanin accumulation and the activation of stress protective mechanisms. PLoS One. 2012;7(5):e37287.

10. de Block M, Verduyn C, de Brouwer D, Cornelissen M. Poly (ADP-ribose) polymerase in plants affects energy homeostasis, cell death and stress tolerance. Plant J. 2005:41(1):95-106.

11. Schreiber V, Dantzer F, Ame JC, de Murcia G. Poly (ADP-ribose): novel functions for an old molecule. Nat Rev Mol Cell Biol. 2006:7(7):517-28.

12. Vyas S, Chesarone-Cataldo M, Todorova T, Huang YH, Chang P. A systematic analysis of the PARP protein family identifies new functions critical for cell physiology. Nat Commun. 2013;4(1):2240.

13. Chen YM, Shall S, O'Farrell M. Poly (ADP-ribose) polymerase in plant nuclei. Eur J Biochem. 1994;224(1):135-42.

14. Amor $Y$, Babiychuk $E$, Inze $D$, Levine $A$. The involvement of poly (ADP-ribose) polymerase in the oxidative stress responses in plants. FEBS Lett. 1998;440(1-2):1-7.

15. Tian R, Zhang GY, Yan CH, Dai YR. Involvement of poly (ADP-ribose) polymerase and activation of caspase-3-like protease in heat shock-induced apoptosis in tobacco suspension cells. FEBS Lett. 2000;474(1):11-5.

16. Willmitzer $L$. Demonstration of in vitro covalent modification of chromosomal proteins by poly (ADP) ribosylation in plant nuclei. FEBS Lett. 1979;108(1):13-6.

17. Babiychuk E, Cottrill PB, Storozhenko S, Fuangthong M, Chen Y, O'Farrell MK van Montagu M, Inze D, Kushnir S. Higher plants possess two structurally different poly (ADP-ribose) polymerases. Plant J. 1998;15(5):635-45.

18. Briggs AG, Bent AF. Poly (ADP-ribosyl) ation in plants. Trends Plant Sci. 2011; 16(7):372-80.

19. Lamb RS, Citarelli M, Teotia S. Functions of the poly (ADP-ribose) polymerase superfamily in plants. Cell Mol Life Sci. 2012;69(2):175-89.

20. Feng B, Liu C, de Oliveira MV, Intorne AC, Li B, Babilonia K, Filho GADS, Shan $L$, He P. Protein poly (ADP-ribosyl) ation regulates arabidopsis immune gene expression and defense responses. PLoS Genet. 2015;11(1):e1004936.

21. Liu C, Wu Q, Liu W, Gu Z, Wang W, Xu P, Ma H, Ge X. Poly (ADP-ribose) polymerases regulate cell division and development in Arabidopsis roots. J Integr Plant Biol. 2017;59(7):459-74. 
22. Song J, Keppler BD, Wise RR, Bent AF. PARP2 is the predominant poly (ADPribose) polymerase in Arabidopsis DNA damage and immune responses. PLoS Genet. 2015;11(5):e1005200.

23. Jia Q, den Dulk-Ras A, Shen H, Hooykaas PJ, de Pater S. Poly (ADPribose) polymerases are involved in microhomology mediated back-up non-homologous end joining in Arabidopsis thaliana. Plant Mol Biol. 2013;82(4-5):339-51.

24. Zhang H, Gu Z, Wu Q, Yang L, Liu C, Ma H, Xia Y, Ge X. Arabidopsis PARG1 is the key factor promoting cell survival among the enzymes regulating posttranslational poly (ADP-ribosyl)ation. Sci Rep. 2015;5(1):15892.

25. Adams-Phillips L, Briggs AG, Bent AF. Disruption of poly (ADP-ribosyl) ation mechanisms alters responses of Arabidopsis to biotic stress. Plant Physiol. 2010;152(1):267-80.

26. Vanderauwera $S$, de Block $M$, de Steene NB, van de Cotte B, Metzlaff $M$, van Breusegem F. Silencing of poly (ADP-ribose) polymerase in plants alters abiotic stress signal transduction. Proc Natl Acad Sci U S A. 2007;104(38): 15150-5.

27. Pham PA, Wahl V, Tohge T, de Souza LR, Zhang Y, Do PT, Olas JJ, Stitt M, Araujo WL, Fernie AR. Analysis of knockout mutants reveals non-redundant functions of poly (ADP-ribose) polymerase isoforms in Arabidopsis. Plant Mol Biol. 2015;89(4-5):319-38.

28. Schulz P, Jansseune K, Degenkolbe T, Meret M, Claeys H, Skirycz A, Teige M, Willmitzer L, Hannah MA. Poly (ADP-ribose) polymerase activity controls plant growth by promoting leaf cell number. PLoS One. 2014;9(2):e90322.

29. Rissel D, Heym PP, Thor K, Brandt W, Wessjohann LA, Peiter E. No silver bullet - canonical poly (ADP-ribose) polymerases (PARPs) are no universal factors of abiotic and biotic stress resistance of Arabidopsis thaliana. Front Plant Sci. 2017;8:59.

30. Rissel D, Losch J, Peiter E. The nuclear protein poly (ADP-ribose) polymerase 3 (AtPARP3) is required for seed storability in Arabidopsis thaliana. Plant Biol. 2014;16(6):1058-64.

31. Hunt L, Holdsworth MJ, Gray JE. Nicotinamidase activity is important for germination. Plant J. 2007:51(3):341-51.

32. Hottiger MO, Hassa PO, Luscher B, Schuler H, Koch-Nolte F. Toward a unified nomenclature for mammalian ADP-ribosyltransferases. Trends Biochem Sci. 2010;35(4):208-19.

33. Langelier MF, Planck JL, Roy S, Pascal JM. Structural basis for DNA damagedependent poly (ADP-ribosyl) ation by human PARP-1. Science. 2012; 336(6082):728-32.

34. Loseva O, Jemth AS, Bryant HE, Schuler H, Lehtio L, Karlberg T, Helleday T. PARP-3 is a mono-ADP-ribosylase that activates PARP-1 in the absence of DNA. J Biol Chem. 2010;285(11):8054-60.

35. Altmeyer M, Messner S, Hassa PO, Fey M, Hottiger MO. Molecular mechanism of poly (ADP-ribosyl) ation by PARP1 and identification of lysine residues as ADP-ribose acceptor sites. Nucleic Acids Res. 2009;37(11):3723-38.

36. Gibson BA, Zhang $Y$, Jiang H, Hussey KM, Shrimp JH, Lin H, Schwede F, Yu $Y$, Kraus WL. Chemical genetic discovery of PARP targets reveals a role for PARP-1 in transcription elongation. Science. 2016;353(6294):45-50.

37. Vodenicharov MD, Ghodgaonkar MM, Halappanavar SS, Shah RG, Shah GM. Mechanism of early biphasic activation of poly (ADP-ribose) polymerase-1 in response to ultraviolet B radiation. J Cell Sci. 2005;118(Pt 3):589-99.

38. Kelley LA, Mezulis S, Yates CM, Wass MN, Sternberg MJ. The Phyre2 web portal for protein modeling, prediction and analysis. Nat Protoc. 2015;10(6):845-58

39. Trott O, Olson AJ. AutoDock vina: improving the speed and accuracy of docking with a new scoring function, efficient optimization, and multithreading. J Comput Chem. 2010;31(2):455-61.

40. Vyas S, Matic I, Uchima L, Rood J, Zaja R, Hay RT, Ahel I, Chang P. Family-wide analysis of poly (ADP-ribose) polymerase activity. Nat Commun. 2014;5:4426.

41. Chen J, Ghorai MK, Kenney G, Stubbe J. Mechanistic studies on bleomycinmediated DNA damage: multiple binding modes can result in doublestranded DNA cleavage. Nucleic Acids Res. 2008;36(11):3781-90.

42. Pascucci B, Russo MT, Crescenzi M, Bignami M, Dogliotti E. The accumulation of MMS-induced single strand breaks in G1 phase is recombinogenic in DNA polymerase beta defective mammalian cells. Nucleic Acids Res. 2005;33(1):280-8.

43. Amé J-C, Rolli V, Schreiber V, Niedergang C, Apiou F, Decker P, Muller S, Höger T, de Murcia JM, de Murcia G. PARP-2, a novel mammalian DNA damage-dependent poly (ADP-ribose) polymerase. J Biol Chem. 1999; 274(25):17860-8
44. Liu C, Vyas A, Kassab MA, Singh AK, Yu X. The role of poly ADP-ribosylation in the first wave of DNA damage response. Nucleic Acids Res. 2017;45(14): 8129-41.

45. Schmutz J, Cannon SB, Schlueter J, Ma J, Mitros T, Nelson W, Hyten DL, Song Q, Thelen JJ, Cheng J, et al. Genome sequence of the palaeopolyploid soybean. Nature. 2010;463(7278):178-83.

46. Haince JF, McDonald D, Rodrigue A, Dery U, Masson JY, Hendzel MJ, Poirier GG. PARP1-dependent kinetics of recruitment of MRE11 and NBS1 proteins to multiple DNA damage sites. J Biol Chem. 2008;283(2):1197-208.

47. Okano S, Lan L, Caldecott KW, Mori T, Yasui A. Spatial and temporal cellular responses to single-strand breaks in human cells. Mol Cell Biol. 2003;23(11): 3974-81.

48. Meng L, Feldman L. A rapid TRIzol-based two-step method for DNA-free RNA extraction from Arabidopsis siliques and dry seeds. Biotechnol J. 2010; 5(2):183-6.

49. Katoh K, Standley DM. MAFFT multiple sequence alignment software version 7: improvements in performance and usability. Mol Biol Evol. 2013; 30(4):772-80.

50. Goodstein DM, Shu S, Howson R, Neupane R, Hayes RD, Fazo J, Mitros T, Dirks W, Hellsten U, Putnam N, et al. Phytozome: a comparative platform for green plant genomics. Nucleic Acids Res. 2012;40(Database issue):D1178-86

51. Ming R, VanBuren R, Liu Y, Yang M, Han Y, Li LT, Zhang Q, Kim MJ, Schatz MC, Campbell M, et al. Genome of the long-living sacred lotus (Nelumbo nucifera Gaertn.). Genome Biol. 2013;14(5):R41.

52. Nystedt B, Street NR, Wetterbom A, Zuccolo A, Lin YC, Scofield DG, Vezzi F, Delhomme N, Giacomello S, Alexeyenko A, et al. The Norway spruce genome sequence and conifer genome evolution. Nature. 2013;497(7451): 579-84

53. Guan R, Zhao Y, Zhang H, Fan G, Liu X, Zhou W, Shi C, Wang J, Liu W, Liang $X$, et al. Draft genome of the living fossil Ginkgo biloba. Gigascience. 2016; 5(1):49.

54. Zerbino DR, Achuthan P, Akanni W, Amode MR, Barrell D, Bhai J, Billis K, Cummins C, Gall A, Giron CG, et al. Ensembl 2018. Nucleic Acids Res. 2018; 46(D1):D754-61

55. Grigoriev IV, Nikitin R, Haridas S, Kuo A, Ohm R, Otillar R, Riley R, Salamov A, Zhao X, Korzeniewski F, et al. MycoCosm portal: gearing up for 1000 fungal genomes. Nucleic Acids Res. 2014:42(Database issue):D699-704.

56. Buchfink B, Xie C, Huson DH. Fast and sensitive protein alignment using DIAMOND. Nat Methods. 2015;12(1):59-60.

57. Kalvari I, Argasinska J, Quinones-Olvera N, Nawrocki EP, Rivas E, Eddy SR, Bateman A, Finn RD, Petrov Al. Rfam 13.0: shifting to a genome-centric resource for non-coding RNA families. Nucleic Acids Res. 2018;46(D1):D335-42.

58. Larsson A. Aliview: a fast and lightweight alignment viewer and editor for large datasets. Bioinformatics. 2014;30(22):3276-8.

59. Capella-Gutierrez S, Silla-Martinez JM, Gabaldon T. trimAl: a tool for automated alignment trimming in large-scale phylogenetic analyses. Bioinformatics. 2009;25(15):1972-3.

60. Stamatakis A. RAxML version 8: a tool for phylogenetic analysis and postanalysis of large phylogenies. Bioinformatics. 2014;30(9):1312-3.

61. Ho SN, Hunt HD, Horton RM, Pullen JK, Pease LR. Site-directed mutagenesis by overlap extension using the polymerase chain reaction. Gene. 1989;77(1):51-9.

\section{Publisher's Note}

Springer Nature remains neutral with regard to jurisdictional claims in published maps and institutional affiliations.

Ready to submit your research? Choose BMC and benefit from:

- fast, convenient online submission

- thorough peer review by experienced researchers in your field

- rapid publication on acceptance

- support for research data, including large and complex data types

- gold Open Access which fosters wider collaboration and increased citations

- maximum visibility for your research: over $100 \mathrm{M}$ website views per year

At $\mathrm{BMC}$, research is always in progress.

Learn more biomedcentral.com/submissions 